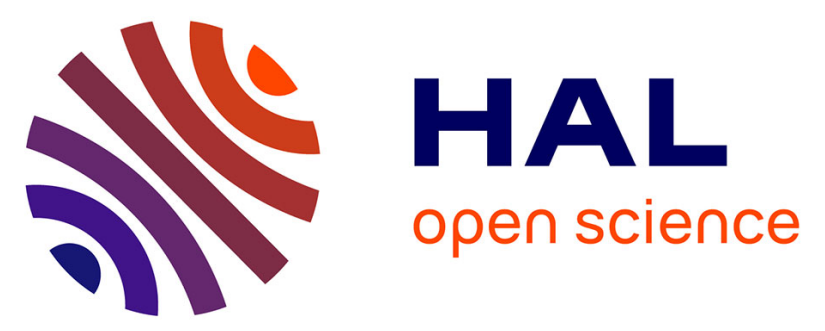

\title{
Conformational maps of human 20S proteasomes reveal PA28- and immuno-dependent inter-ring crosstalks
}

\author{
Jean Lesne, Marie Locard-Paulet, Julien Parra, Dušan Zivković, Thomas
}

Menneteau, Marie-Pierre Bousquet, Odile Burlet-Schiltz, Julien Marcoux

\section{- To cite this version:}

Jean Lesne, Marie Locard-Paulet, Julien Parra, Dušan Zivković, Thomas Menneteau, et al.. Conformational maps of human 20S proteasomes reveal PA28- and immuno-dependent inter-ring crosstalks. Nature Communications, 2020, 10.1038/s41467-020-19934-z . hal-02994942v2

\section{HAL Id: hal-02994942 \\ https://hal.science/hal-02994942v2}

Submitted on 22 Oct 2021

HAL is a multi-disciplinary open access archive for the deposit and dissemination of scientific research documents, whether they are published or not. The documents may come from teaching and research institutions in France or abroad, or from public or private research centers.
L'archive ouverte pluridisciplinaire HAL, est destinée au dépôt et à la diffusion de documents scientifiques de niveau recherche, publiés ou non, émanant des établissements d'enseignement et de recherche français ou étrangers, des laboratoires publics ou privés. 
ARTICLE

\title{
Conformational maps of human 205 proteasomes reveal PA28- and immuno-dependent inter-ring crosstalks
}

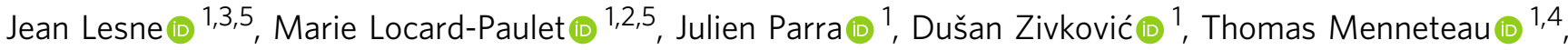 \\ Marie-Pierre Bousquet ${ }^{1}$, Odile Burlet-Schiltz ${ }^{1} \&$ Julien Marcoux (D) ${ }^{1 凶}$
}

Hydrogen-Deuterium eXchange coupled to Mass Spectrometry (HDX-MS) is now common practice in structural biology. However, it is most of the time applied to rather small oligomeric complexes. Here, we report on the use of HDX-MS to investigate conformational differences between the human standard 20S (std20S) and immuno 20S (i20s) proteasomes alone or in complex with PA28 $\alpha \beta$ or PA28 $\gamma$ activators. Their solvent accessibility is analyzed through a dedicated bioinformatic pipeline including stringent statistical analysis and 3D visualization. These data confirm the existence of allosteric differences between the std20S and i20S at the surface of the $\alpha$-ring triggered from inside the catalytic $\beta$-ring. Additionally, binding of the PA28 regulators to the $20 \mathrm{~S}$ proteasomes modify solvent accessibility due to conformational changes of the $\beta$-rings. This work is not only a proof-of-concept that HDX-MS can be used to get structural insights on large multi-protein complexes in solution, it also demonstrates that the binding of the std2OS or i20S subtype to any of its PA28 activator triggers allosteric changes that are specific to this 20S/PA28 pair.

\footnotetext{
${ }^{1}$ Institut de Pharmacologie et de Biologie Structurale (IPBS), Université de Toulouse, CNRS, UPS, Toulouse, France. ${ }^{2}$ Novo Nordisk Foundation Center for Protein Research, University of Copenhagen, Copenhagen, Denmark. ${ }^{3}$ Present address: Centre de Biologie Structurale, CNRS, Université de Montpellier, INSERM, 34090 Montpellier, France. ${ }^{4}$ Present address: Institute of Structural and Molecular Biology, Division of Biosciences, University College London, London WC1E 6BT, UK. ${ }^{5}$ These authors contributed equally: Jean Lesne, Marie Locard-Paulet. ${ }^{凶}$ email: julien.marcoux@ipbs.fr
} 
$\mathrm{T}$ he ubiquitin proteasome system (UPS) is central to proteostasis. Its most downstream element is the $26 \mathrm{~S}$ proteasome that clears the cells of abnormal, denatured, or damaged proteins and regulates degradation of short-lived proteins, in most cases conjugated to ubiquitin. The $26 \mathrm{~S}$ proteasome is a highly conserved compartmentalized multicatalytic protease composed of more than 30 subunits constituting the 20 S catalytic core and the 195 regulatory complex ${ }^{1}$. Its activity is directly involved in many cytokines and hub proteins intracellular concentration $^{2}$, and regulates immunogenic peptide production ${ }^{3}$. It is the focus of intense regulation and dynamically localizes within cells in response to physiological and external perturbations ${ }^{4,5}$ and its genetic polymorphism is directly responsible for numerous pathologies including cancer, heart disease, and type 2 diabetes to name a few ${ }^{6,7}$.

The main mechanism regulating the proteasome activity is the substitution of the catalytic subunits $\beta 1, \beta 2$, and $\beta 5$, respectively harboring caspase-like, trypsin-like, and chymotrypsin-like activities, and constituting the standard 20S (std20S) with other subunits. For example, the immunoproteasome (i20S) contains the immuno-subunits $\beta 1 i, \beta 2 i$, and $\beta 5 i$ that have different cleavage specificities and generate immunogenic peptides ${ }^{8}$. It is strongly expressed in immune cells and can be induced in most tissues by interferon- $\gamma$.

The proteolytic activity of the $20 \mathrm{~S}$ is also regulated by interacting proteins, the most common being the ATP-dependent $19 \mathrm{~S}$ activator involved in the UPS degradation pathway. However, less abundant ubiquitin-independent regulators such as PA28a $\beta$, PA28 $\gamma$, or PA200 also activate the 20 S and modify its substrate specificity. In addition, a vast collection of Proteasome Interacting Proteins (PIPs) regulate or assist proteasomal functions ${ }^{9-16}$.

Besides its subunit composition and association with regulators and/or PIPs, the proteasome is subjected to a wide variety of posttranslational modifications (PTMs) affecting its subunits activity ${ }^{17-19}$. The combination of these three levels of regulation (catalytic subunits, regulators, PTMs) implies a wide proteasome subtype heterogeneity, which probably results in specialized functions that can adapt protein degradation pathways to changing conditions in the cell. One of today's main challenges is to understand how different proteasome subtypes influence its proteolytic activity and the cell function.

The proteasome has been intensely studied from a structural and functional point of view since its discovery in $1988^{20}$. Electron microscopy (EM) allowed to observe the 20S in complex with different regulators ${ }^{21-26}$ together with certain catalytic intermediate-states of the $26 \mathrm{~S}^{27}$. Covalent cross-linking coupled to mass spectrometry (MS) helped refining $26 \mathrm{~S}$ structures ${ }^{28,29}$ and generating structural models involving different partners, including the $19 \mathrm{~S}^{23}, \mathrm{Ecm} 29^{15}$, Ubp6 ${ }^{30}$, and other $\mathrm{PIPs}^{15}$. X-ray crystallography also provided high-resolution structures of proteasomes alone or in the presence of covalent inhibitors ${ }^{31}$, regulators $^{32,33}$, and complexes of proteasome with their associated regulators ${ }^{15,31,32}$.

The complex size is obviously a limit for its analysis by Nuclear Magnetic Resonance (NMR). However, the structure of the eukaryotic proteasome from Thermoplasma acidophilum, containing only one type of subunit $\alpha$ and $\beta$, has been resolved. It showed evidence of a remodelling of the catalytic sites located in the center of the proteasome upon binding of a regulatory particle on its surface, which can be described as an outer-to-inner allosteric change ${ }^{34}$. A reverse inner-to-outer change was also observed at the binding interface when modifying the catalytic site, but this was not confirmed by the comparison of the std20S $\mathrm{X}$-ray structure ${ }^{35}$ with the recent i20S X-ray ${ }^{36}$ and cryo- $\mathrm{EM}^{37}$ structures (RMSD $=0.392$ and $0.480 \AA$, respectively). However, the outer-to-inner mechanism was recently shown in the human
20 cryo-EM structure upon PA200 binding ${ }^{25}$ : conformational changes were found not only at the binding interface (opening of the pore), but also down to the catalytic sites.

Despite these achievements, high-resolution methods are still limited by either the size, the heterogeneity, or the dynamics of the purified complexes. This is evidenced by the small number of structures of the $20 \mathrm{~S}$ bound to regulators and their numerous unresolved regions.

Here, we optimized the emerging method HydrogenDeuterium eXchange coupled to MS (HDX-MS) to investigate conformational changes occurring upon binding of the std/i20S to PA28 regulators. This method, developed in the $90 \mathrm{~s}$, enables the detection of conformational differences between two protein samples. Briefly, the proteins or protein complexes of interest are diluted in a deuterated buffer and hydrogen atoms from the peptide bonds are exchanged with the deuterium atoms of the solution. The rate of exchange of each amide hydrogen depends on its solvent accessibility and involvement in the stabilization of secondary structures: regions that are relatively more solventaccessible or flexible present a higher deuteration rate than the ones that are hidden in the core of the protein or rigid. This expanding method can thus be used to study protein dynamics ${ }^{38}$, compare protein conformations (in different buffer conditions, after mutation, ligand binding ${ }^{39}$, or to identify protein-protein or protein-ligand binding interfaces ${ }^{40,41}$. We present the HDXMS analysis of the entire human std and i20S core particles as well as their PA28a $\beta$ and PA28 $\gamma$ regulators, mapping solvent accessibility/dynamics for each complex. The conformational maps of the std and i20S present significant differences, and their differential analysis with and without regulators provide a molecular rationale for their distinct functions. Our data provide evidence for the human $20 \mathrm{~S}$ inner-to-outer allosteric change upon incorporation of the immuno-subunits and the reverse outer-toinner transduction signal upon PA28 binding, illustrating the interplay between the different proteasome regulation pathways. Altogether, this work highlights the potential of HDX-MS to generate low resolution but informative structural information on large hetero-oligomeric complexes. It opens the door to many other applications, including identifying PIPs binding surfaces and the stabilizing/destabilizing effects of other regulators of the 20 S activity, including small molecules.

\section{Results}

Dynamic interfaces uniting the four rings of the $20 S$ proteasome. HDX consists in the exchange of backbone amide hydrogens $(H)$ with deuteriums $(D)$ in proteins in solution. The exchange rate depends on the solvent accessibility and/or the flexibility of a given region, thereby providing low resolution but crucial information on protein conformation. We performed two distinct analyses (Fig. 1a): (i) identification of the most accessible and/or dynamic regions of the proteins in solution, and (ii) differential analysis of each protein alone or in complex. We monitored the incorporation of deuterium for each sample from 0 to $30 \mathrm{~min}$ in triplicate experiments (Fig. 1b). For differential analyses, we compared the peptide relative deuterium uptakes (RDU) of the proteins alone vs. in presence of their binding partner. The peptides were considered significantly different when they presented a $P$ value of the ANOVA $\leq 0.01$ after correction for multiple testing (Benjamini-Hochberg) and three successive time points with an absolute difference between the two conditions $>4$ times the experimental standard deviation observed in the dataset (Fig. 1c) (see Methods for more detailed information). The peptide-level RDUs or differences of RDU were mapped to the protein sequences as described in Fig. 1d: when several peptides covered the same region of a protein we 


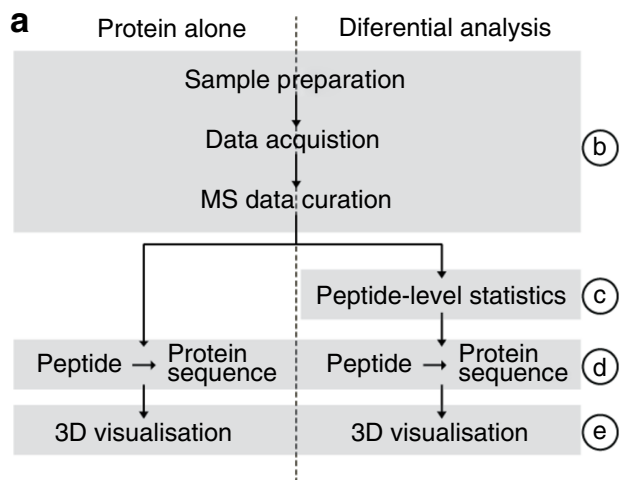

b

C
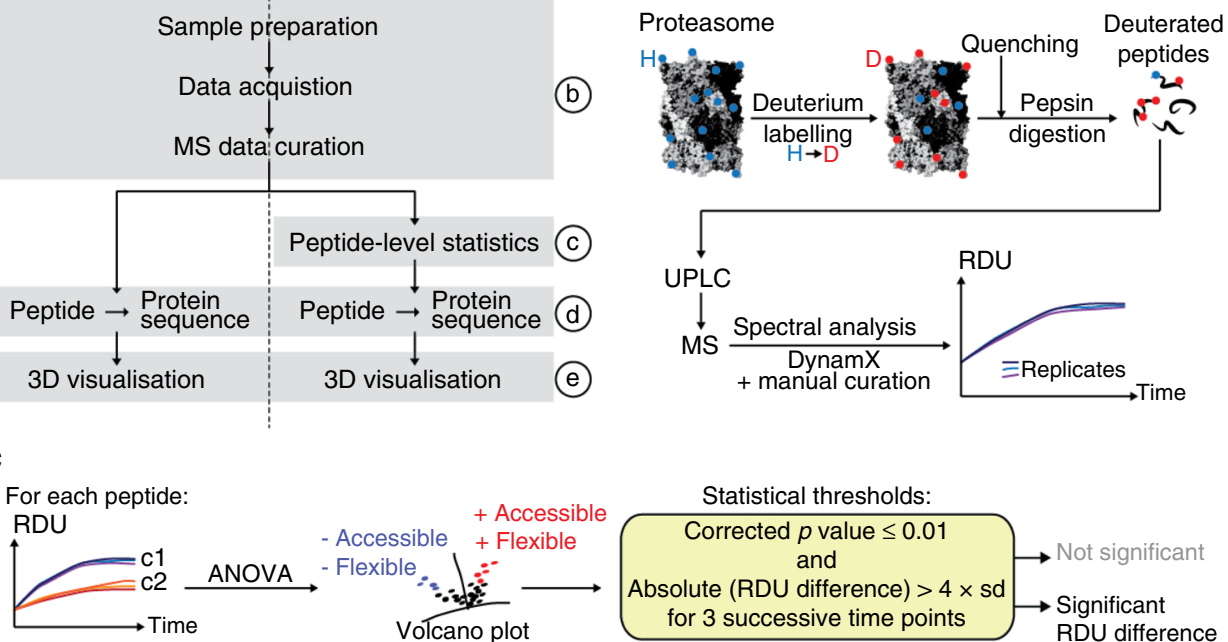

Statistical thresholds:

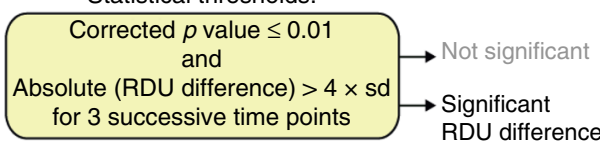

d

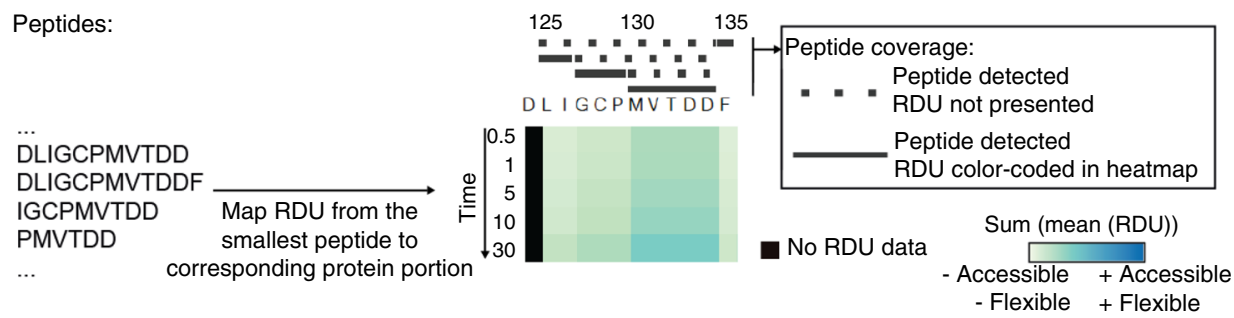

e

Protein alone

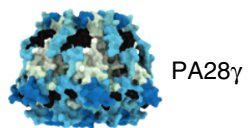

Sum (mean (RDU))

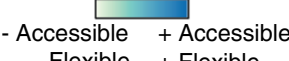

- Flexible + Flexible

- No RDU data
Differential analysis

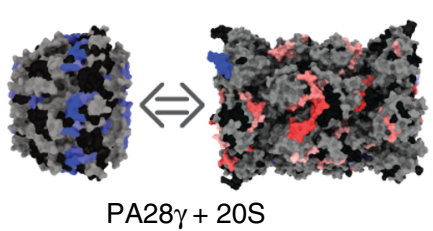

Sum (mean (diff. RDU))

in complex alone

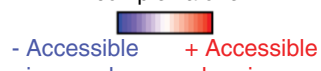

when in complex when in complex

no RDU data

Not significantly regulated

Fig. 1 HDX-MS workflow applied to the analysis of the 20 s proteasome and its regulators. a Overview of the workflows applied to the analysis of proteins alone (left) or differential analysis of proteins alone vs. in complex (right). The different steps are described in more detail in $\mathbf{b}$-e as referred to on the right side. $\mathbf{b}$ Sample preparation and MS data acquisition. Proteins (alone or in complex) were subjected to hydrogen (blue points) to deuterium (red points) exchange in triplicate experiments for $0,0.5,1,5,10$, and 30 min before being subjected to pepsin digestion. Peptides were then separated by liquid chromatography (UPLC) and analysed by MS. Spectra were curated using DynamX before statistical analysis and/or mapping of their RDU to the protein sequence (consolidation). c Statistical comparison of proteins alone vs. in complex: kinetics of solvent accessibility were compared for each peptide using ANOVA. Peptides with a Benjamini-Hochberg corrected $P$ value $\leq 0.01$ and 3 successive time points presenting an absolute difference of RDU $>4$ times the mean standard deviation between replicates in the entire dataset were considered regulated and colored in the final figures. Otherwise, peptides were colored in gray in the heatmaps and 3D figures of differential analyses. $\mathbf{d}$ RDUs of the smallest peptides were mapped to the amino-acid portion they cover on the protein sequence. These were then presented as color gradients on heatmaps: light green and dark blue correspond to low and high RDU, respectively. The origin of RDU reported on the heatmap is indicated above the protein sequence with peptides represented as solid and dashed lines when their RDUs were reported or ignored, respectively. Residue numbers are indicated on the top. e 3D visualization of the RDU (left panel) or significant differences of RDU (right panel) measured in this study: blue and red correspond to a reduction or an increase of RDU in the complex, respectively. The mapping of peptide-level data to protein sequence was performed as described in $\mathbf{d}$ with the sum of RDU per timepoint (sum(mean(RDU))) or the sum of the mean of difference of RDU per timepoint (excepted TO) (sum(mean(diff. RDU))). H hydrogen, D deuterium, diff. difference, RDU relative deuterium uptake.

kept only the RDU (or difference of RDU) of the shortest one. Finally, we mapped the RDUs to the protein $3 \mathrm{D}$ structures in order to visualize the regions the most accessible to solvent (Fig. 1e, left panel), or presenting significant differences of deuteration (Fig. 1e, right panel). For differential analysis, we colored the $3 \mathrm{D}$ structures with the sum of RDU differences between the two conditions (the values per timepoint are available in Supplementary Data 1-3). In this context, modification of solvent accessibility upon complex formation can be due to the presence of a binding interface or to allosteric changes. This approach was employed to identify the conformational differences between the std20S and i20S, and to compare their structural changes upon 
a

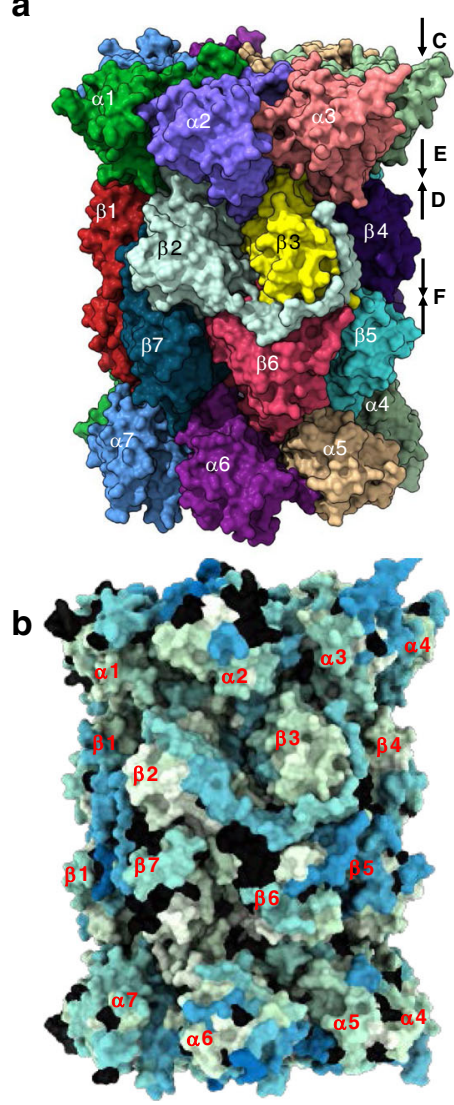

c

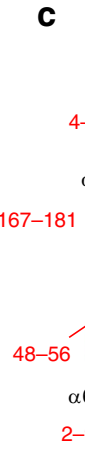

c

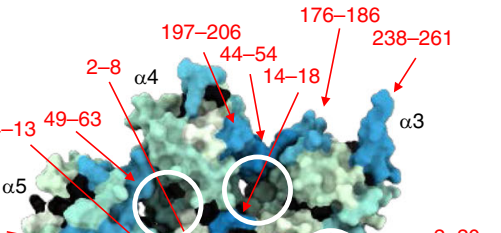

e

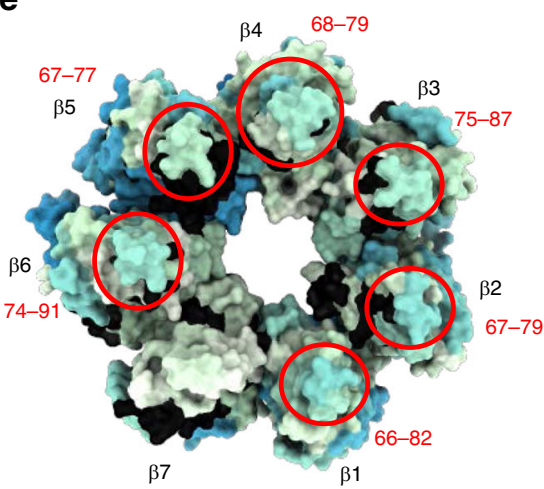
$\beta 7$

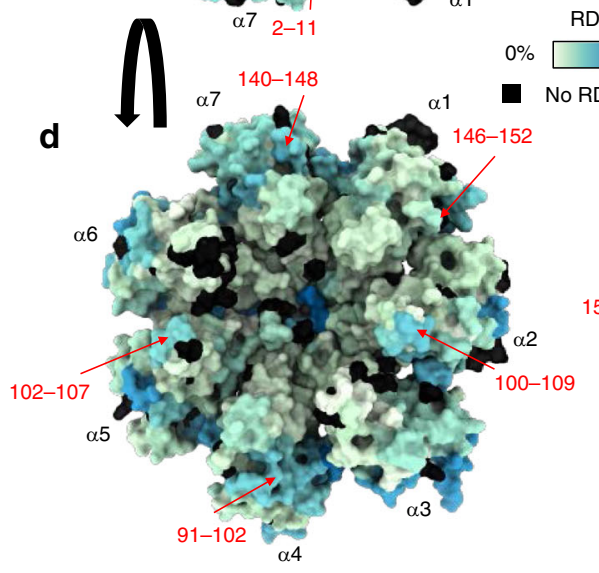
${ }_{194-202}^{\alpha 2}$ $5^{194-202}$ DU

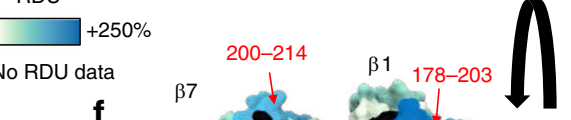

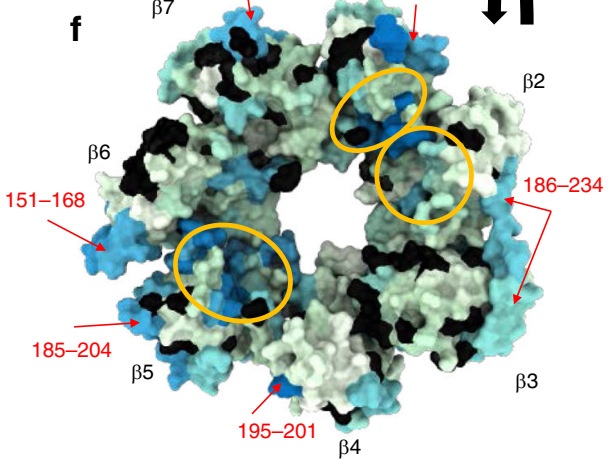

Fig. 2 HDX-MS of the std20S reveals a dynamic pore entrance and inter-ring interface. a, b Structure of the human std20S proteasome (PDB: $5 L E 5)^{35}$ showing the four stacked $\alpha \beta \beta \alpha$ heptameric rings colored by subunit (a) or relative deuterium uptake (RDU) (b). c-f Their solvent accessibility is represented on each face of the $\alpha$-ring (solvent interface (c) and $\beta$-ring interface (d)) and $\beta$-ring ( $\alpha$-ring interface (e) and $\beta$-ring interface (f)) as the sum of consolidated relative deuterium uptakes between 0.5 and 30 min (described in Fig. 1b, c). The $\alpha$-pockets (docking sites for the C-termini of PA28 regulators) are circled in white in $\mathbf{c}$. Regions discussed in the text are indicated with red arrows and the moderately flexible/accessible bulges at the interface between the $\alpha$ - and $\beta$-ring are circled in red in $\mathbf{e}$. The corresponding residues are indicated in red around the structures. Active sites are circled in orange (f). The ring faces visualized in $\mathbf{d}-\mathbf{f}$ are indicated by arrows on the right of the structure in $\mathbf{a}$.

binding to PA28 $\alpha \beta$ or PA28 $\gamma$. For this, we purchased commercial i20S purified from human spleen previously used for in vitro proteolytic assays $s^{42-44}$.

We obtained $89 \%$ subunit sequence coverage on average for the 17 subunits of the std/i20S (Supplementary Fig. 1, Supplementary Data 4 and 5, Supplementary Table 1). HDX-MS results were mapped on the 3D structure of the human std20S (PDB: 5LE5, Fig. 2a, b). As expected, we found a faster and higher deuteration of the solvent-facing $\alpha$-ring interface of the std20S compared to any other ring interfaces (Fig. 2c). This observation benchmarks our approach by confirming that high RDUs directly indicate regions of the proteasome that are more dynamic and potentially accessible for interaction with regulators or PIPs. The N-terminal ( $\mathrm{N}$-ter) part of the $\alpha$-subunits constituting the $\alpha$-ring were highly deuterated (2-22 in $\alpha 1,2-20$ in $\alpha 2,14-18$ in $\alpha 3,2-8$ in $\alpha 4,4-13$ in $\alpha 5,2-9$ in $\alpha 6$, and $2-11$ in $\alpha$, Fig. $2 c)$. These $\mathrm{N}$-ter are located around the proteasome pore entry, and their high RDU strongly supports the hypothesis of a flexible pore entrance in the std20S that fluctuates between open and closed states ${ }^{45-47}$. Furthermore, the $\alpha$-ring presented very dynamic patches consisting of noncontiguous peptides from the same subunit in $\alpha 3$ (176-186/ 238-261), a4 (44-54/197-206), and a5 (49-63/167-181) (Fig. 2c). These may interact with many different PIPs. Our analysis also identified dynamic patches at the interface between different subunits like $\alpha 1$ (2-22/165-178) and a2 (194-202), a3 (14-18) and a4 (44-54), and $\alpha 5(167-181)$ and a6 (48-56) that correspond to the 1-2, 3-4, and 5-6 a-pockets ${ }^{48}$ (Fig. 2c, circled in white) known to accommodate the C-terminal (C-ter) tails of Rpt3, Rpt2, and Rpt5, respectively ${ }^{23,48}$. We also identified flexible patches on the interface of the $\alpha$-ring facing the $\beta$-ring, where amino-acid stretches of $\alpha 1$ (146-152), a4 (91-102), a5 (102-107), and $\alpha 7(140-148)$ were slightly more flexible than the remaining of the interface (Fig. 2d and Supplementary Data 3, 6).

The $\beta$-ring was globally less deuterated than the $\alpha$-ring (Fig. 2e, f), which was expected since it does not present a large solvent interface. However, after $30 \mathrm{~min}$, some $\alpha$-facing bulges were moderately deuterated in all $\beta$ subunits but $\beta 7$ : these include the loops between $\alpha$-helices 1 and 2 of $\beta 1$ (66-82), $\beta 2$ (67-79), $\beta 3$ (75-87), $\beta 4$ (68-79), $\beta 5$ (67-77), and $\beta 6$ (74-91) (red circles in Fig. 2e, and Supplementary Data 6). Interestingly, some of these dynamic loops face the highly deuterated regions of the $\alpha$-ring described above and define flexible regions of the $\alpha$-ring/ $\beta$-ring interface. The most dynamic regions of the $\beta$-ring were located on its outer surface and were constituted of the C-ter of $\beta 1$ (178-203), $\beta 2$ (186-234), $\beta 4$ (195-201), $\beta 5$ (185-204), $\beta 6$ (151-168), and $\beta 7$ (200-214) (Fig. 2f in red and Supplementary Data 6). These regions are particularly interesting since they can potentially interact with the numerous PIPs described in the literature.

An inner-to-outer conformational change upon substitution of standard to immuno-subunits. Although mouse i20S and std20S 
possess very similar $\beta 2$ and $\beta 2 \mathrm{i}$ substrate binding channels, there are structural differences between their standard and immunocatalytic subunits $\beta 1$ and $\beta 5$. The S1 and S3 substrate pockets are smaller in $\beta 1 \mathrm{i}$ vs. $\beta 1, \beta 5 \mathrm{i}$ possesses smaller S2 and S3 pockets, and the $S 1$ pocket is larger in $\beta 5 \mathrm{i}$ than in $\beta 5^{31}$. However, very little structural difference is found between the $\alpha$ and noncatalytic $\beta$ subunits of the i20S and std20S (Root-Mean Square Deviation of the $C a<0.72 \AA$ ). The same is true when comparing the recent structure of the human $120 S^{36}$ with the std20S 35 (RMSD of the $\mathrm{C} a<0.67 \AA)$.

The std20S catalytic chamber-localized at the $\beta$-ring/ $\beta$-ring interface-was locally very flexible in our data (Fig. $2 \mathrm{f}$ and Fig. 3). The residues forming the S1 and S2 pockets ${ }^{31}$ of $\beta 1$ were highly deuterated, together with the T23 of the S3 pocket (Fig. $3 \mathrm{~b}$, see Supplementary Data 4 for full kinetics). The catalytic residues of $\beta 2$ were poorly deuterated compared to the remainder of this subunit (Fig. 3c), with the exception of $45-53$ constituting its S1 and S2 pockets. Conversely, most of the residues forming $\beta 5$ catalytic site and substrate pockets were highly deuterated (Fig. $3 \mathrm{~d}$ ). The relatively poor sequence coverage that we obtained for $\beta 2 \mathrm{i}$ limits its analysis and we cannot comment on the accessibility of its S1, S2, and S3 pockets (Fig. 3b and Supplementary Table 1). The direct comparison of RDUs between the standard and immuno-catalytic subunits $(\beta 1 / \beta 1 \mathrm{i}, \beta 2 / \beta 2 \mathrm{i}$, and $\beta 5 / \beta 5 \mathrm{i})$ was not possible due to their sequence differences. Nevertheless, we could acquire the deuteration profiles of the peptides ADSRATAGAY (16-25) of $\beta 5$ and AVDSRASAGSY $(15-25)$ of $\beta 5$ i that cover the same portion of the two subunits. Their difference of deuteration profiles indicate that these residues are more flexible in std20S than in the i20S (Fig. $3 \mathrm{~d}$ and Supplementary Data 4).

Allosteric differences beyond the catalytic subunits were shown in the prokaryotic std and i20S analysed by $\mathrm{NMR}^{34}$. These were not observed in mouse crystallographic structures ${ }^{31}$, may be due to crystal packing. In order to confirm or infirm this inner-toouter allosteric change in human, we compared the RDUs of all the noncatalytic subunits of the i20S (Supplementary Data 4-6) with those of the std20S (as presented Fig. 1).

The introduction of immuno-catalytic subunits resulted in significant conformational changes on the noncatalytic $20 \mathrm{~S}$ subunits. The results of our statistical analysis are presented in Fig. 4, and Supplementary Data 1, 4, 7-10. Overall, the noncatalytic $\beta$-ring subunits were more dynamic in the i20S than in the std20S, $(>50$ peptides significantly more deuterated, Fig. $4 \mathrm{a}-\mathrm{b}$ in red, Supplementary Data 1,7$)$. The $\beta$-ring/ $\beta$-ring interface and the channel were particularly more dynamic/ accessible in the i20S vs. std20S (Fig. 4a/e and Supplementary Data 1), together with two $\alpha$-facing bulges of $\beta 6$ and $\beta 7$ (Fig. $4 \mathrm{~b}$, red circles). Four $\beta$-facing bulges of $\alpha 2 / 3 / 5 / 6$ were also significantly more dynamic in the i20S vs. std20S (Fig. 4c, red circles, corrected $P$-values and RDU differences are available in Supplementary Data 9 and 10). The pore entrance on the $\alpha$-ring solvent-accessible surface (a subunits $\mathrm{N}$-ter) was significantly more dynamic/accessible upon replacement of $\beta 1 / 2 / 5$ by the immuno-subunits (Fig. 4d red circles, Supplementary Data 1, 7). This suggests a long-range mechanism enabling the i20S pore to dwell longer in the open-state, and could explain its generally higher activity 49 as well as the various regions from the antechambers that were more accessible in the i20S vs. std20S (98-99 in $\alpha 2 ; 102-109$ in $\alpha 3 ; 128-142$ in $\alpha 5 ; 100-108$ in $\alpha 6$; 95-105 in $\beta 3$; 94-102 in $\beta 4$, Fig. 4e, corrected $P$-values and RDU differences are available in Supplementary Data 9 and 10). Conversely, three solvent-facing regions of the a-ring were significantly more dynamic/accessible in the std20S than in the i20S (Fig. 4d in blue, Supplementary Data 4, 7). Since these constitute potential binding sites for the proteasome regulators, this could explain the preferential association of specific regulators to different $20 \mathrm{~S}$ subtypes. Interestingly, these three subunits also contain regions that are more accessible in the std $20 \mathrm{~S}$ on the $\beta$-ring facing interface (Fig. 4c), suggesting that this dynamic behavior can be related to the presence of the standard catalytic subunits within the $\beta$-ring. More precisely, the a1 102-114 (corrected $P$ value $=5.87 \cdot 10^{-11}$, sum of difference $=$ $-12 \%$ ) and $146-152$ (corrected $P$ value $=2.54 .10^{-7}$, sum of difference $=-12 \%$ ), which were more deuterated in the std20S vs. i20S, are in direct contact with a portion of $\beta 2 / \beta 2 \mathrm{i}(69-72)$ also more accessible in the std20S (Supplementary Data 1). Altogether, these results confirm the inner-to-outer allosteric effect following incorporation of the immuno- $\beta$ subunits that lead to $\alpha$ ring remodeling: more dynamic pore entrance but protected external anchor regions ( $\alpha 3 \mathrm{C}$-ter).

The activation loop of PA28 $\beta$ is less dynamic/accessible than PA28a/ $\gamma$ 's. There is no structure yet for PA28 $\gamma$ but those of the human homoheptameric PA28a (nonphysiological) and murine heteroheptameric PA28a $\alpha_{4} \beta_{3}$ were resolved in $1997^{32}$ and $2017^{33}$, respectively. These monomers are composed of a four-helix bundle, assembled as a barrel-shaped heptamer (Fig. 5) that controls proteasome catalytic activity through a conserved activation loop ${ }^{50,51}$.

The structures of PA28 $\alpha$, PA28 $\beta$, and PA28 $\alpha \beta$ miss a region of 15-31 residues bridging the $\alpha$-helices 1 and 2 , although they were present in the recombinant constructs (Fig. 5a-c and Supplementary Data 11 in red). These apical loops, most likely flexible, sit on top of the regulator and might thereby control the substrate access to the proteasome. The human PA28 $\gamma$ chain has a similar loop (43 residues), and no information on its structure has ever been reported. Since the sequence identity of human PA28 $\gamma$ is closer to PA28a (40\%) than PA28 $\beta$ (35\%) or Plasmodium falciparum (Pf) PA28 (34\%) (Supplementary Table 2), we generated a homology model of human PA28 $\gamma$ (Fig. 5e) using the structure of human PA28 $\alpha^{33}$ as a template with the SWISSMODEL server ${ }^{52}$. The sequence of $P f$ is used as a comparison here because it is part of the very few structures of PA28 regulator homologues available in the PDB with close homology to PA28 $\gamma$ and whose structure was recently solved in complex with the $20 S^{24}$

The sequence coverages obtained upon digestion were above $90 \%$ for all three PA28 subunits across all conditions (Supplementary Fig. 1, Supplementary Data 11, 12 and Supplementary Table 1). Their deuteration pattern revealed similar dynamics for both PA28 $\alpha \beta$ and PA28 $\gamma$ with a very strong protection of most residues inside the channel (Fig. 5d, e and Supplementary Data 11). The missing apical loops (Supplementary Data 11, red rectangles) were very quickly deuterated, a sign of high accessibility/flexibility, that could explain their absence in known structures. The proteasome-facing interface (unstructured $\mathrm{N}$ - and C-termini and activation loops) (Fig. 5d, e, right) and the entrance of the channel (Fig. 5d, e, left) were also strongly deuterated. Remarkably, the regions involved in the binding (Cter) and activation of the proteasome were very accessible/ dynamic when both regulators were alone in solution. However, the activation loop was less deuterated in PA28 $\beta$. The reason why eukaryoric homoheptameric PA28 regulators have evolved towards more complex heteroheptameric PA28 $\alpha \beta$ is not well understood. Given the overall similar tridimensional structures of PA28 $\alpha \beta$ and PA28 $\gamma$, the poorer flexibility observed on PA28 $\beta$ activation loop could, at least partially, explain their different functions. 
a

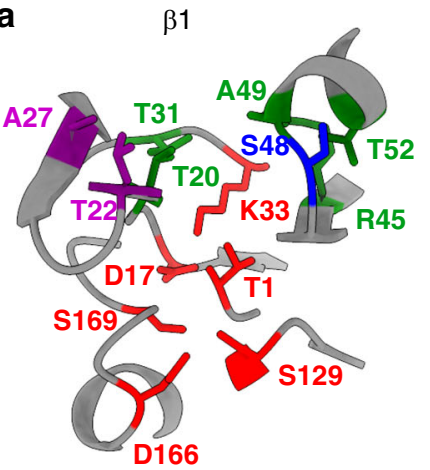

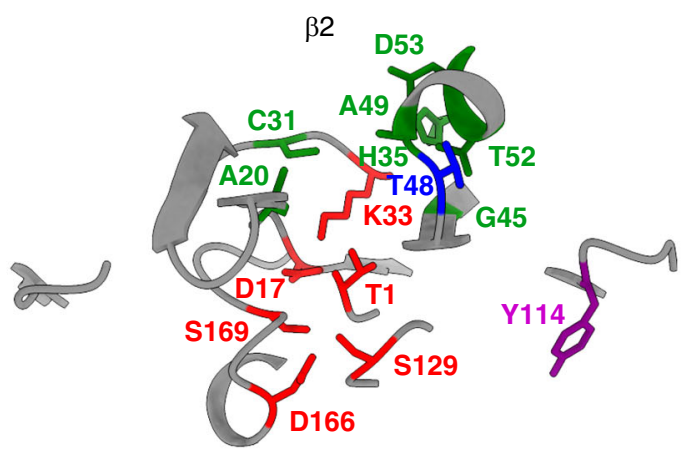

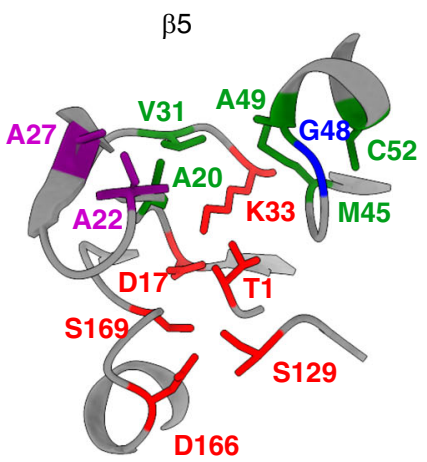

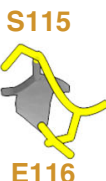

E116

b
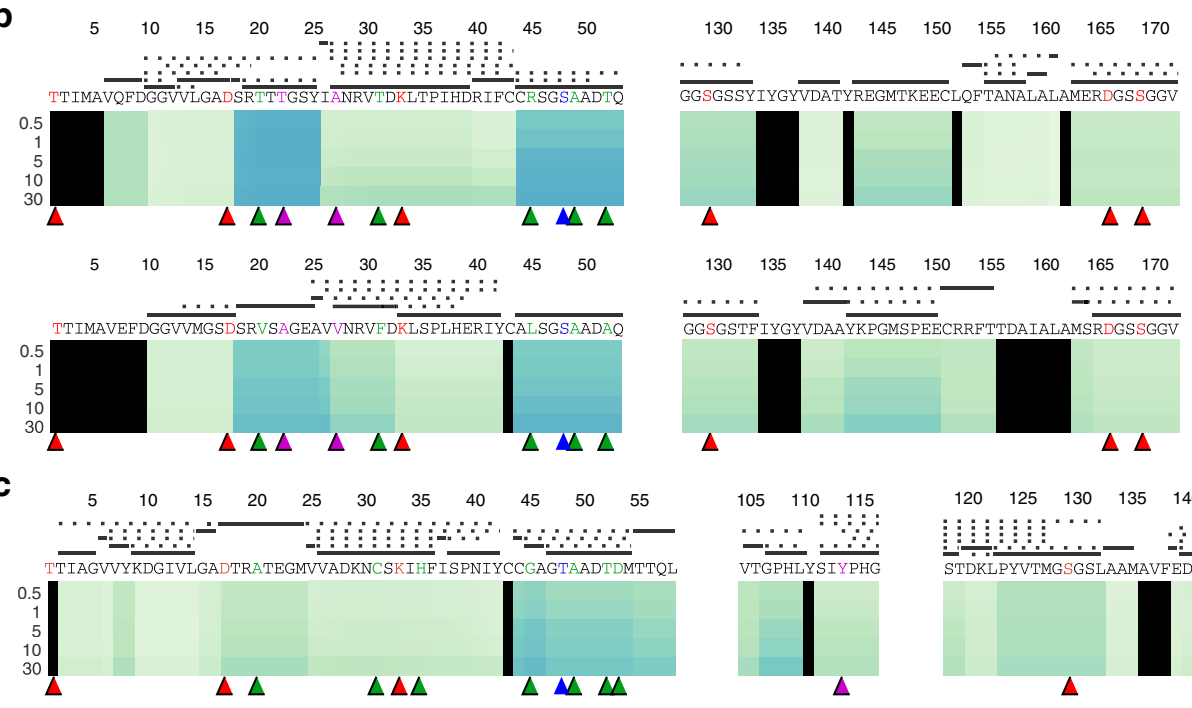

$\beta 1$

Active site

S1 pocket

S2 pocket

$\square$ s3 pocket

$\square$ S' pocket

No RDU data

RDU

$\beta 1 \mathrm{i}$

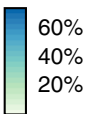

$\Delta$

C
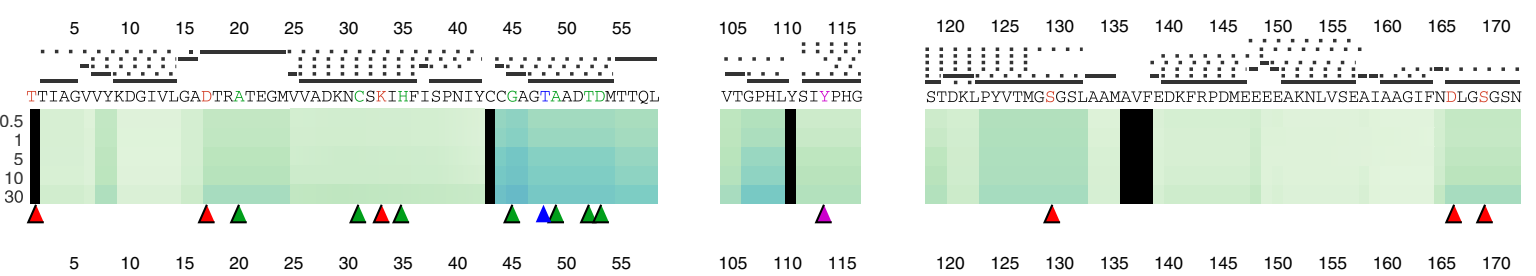

$\begin{array}{lllllllllll}120 & 125 & 130 & 135 & 140 & 145 & 150 & 155 & 160 & 165 & 170\end{array}$ $\underset{\text { TTIAGLVFQDGVILGADTRATNDSVVADKSCEKIHFIAPKIYCCGAGVAADAEMTTRM }}{\cdots}$
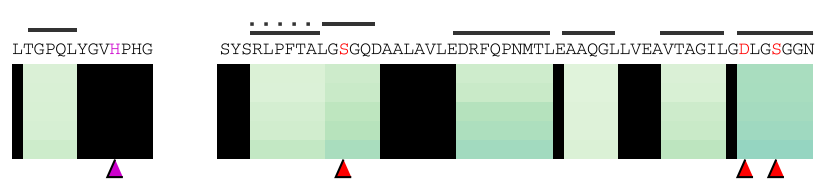

d
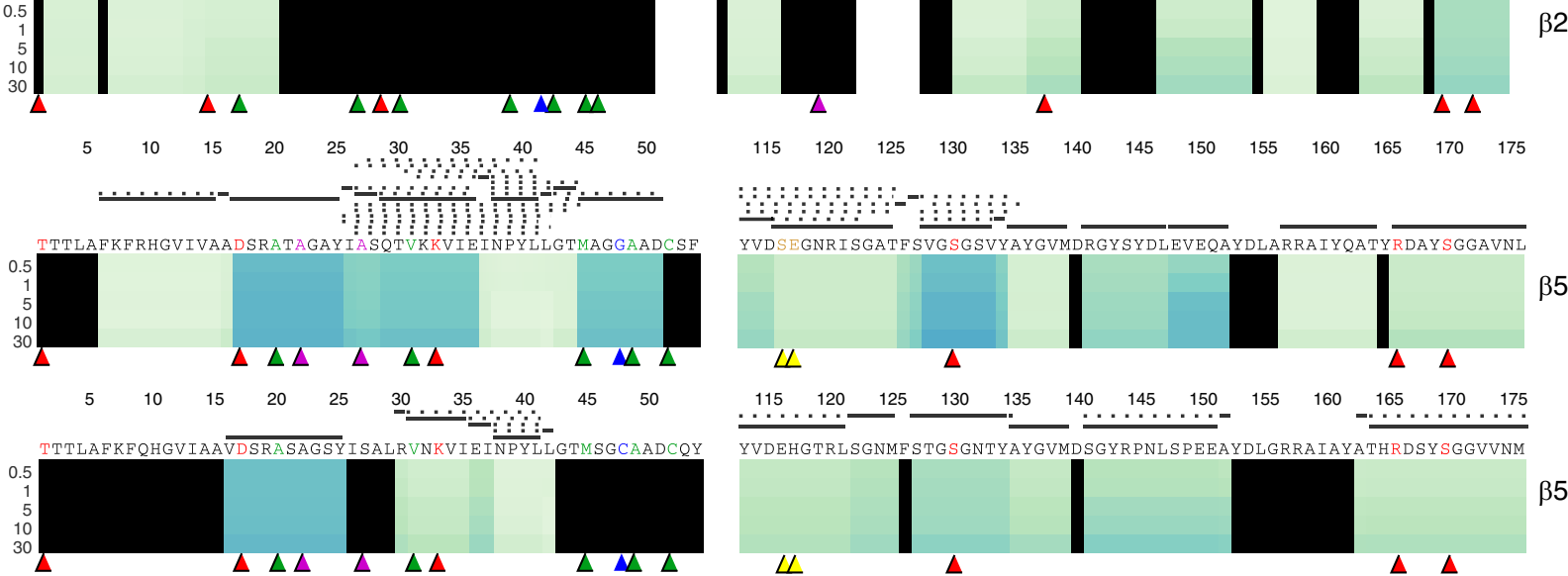

$\beta 5 i$

Fig. 3 Comparison of the catalytic-subunit solvent accessibilities. a Representation of the three catalytic subunits $\beta 1$ (left), $\beta 2$ (middle), and $\beta 5$ (right) of the std2OS (PDB: 5LE5 ${ }^{35}$ ). The residues forming the active site and the substrate-specific pockets are indicated and color-coded (active site, S1, S2, S3, and $\mathrm{S}^{\prime}$ are represented in red, green, blue, purple, and yellow, respectively). b-d Deuteration heatmaps showing the relative deuterium uptake (RDU) for each timepoint of the kinetics, along the protein sequences of $\beta 1 / \beta 1 i(\mathbf{b}), \beta 2 / \beta 2 i(\mathbf{c})$, and $\beta 5 / \beta 5 i(\mathbf{d})$. The peptide sequence coverage is presented above the heatmaps as explained in Fig. 1d. For the sake of clarity, only the sequence stretches containing residues of the active sites and substrate pockets are represented here.

Allosteric changes between PA28 $\gamma$ and PA28a $\beta$ upon $20 S$ binding. Before comparing the PA28 regulators alone and in complex with the std20S, we confirmed their ability to increase the proteasome activity in vitro. In our hands, a 2 -fold molar excess of any of the PA28 regulators doubled the std20S chymotrypsin-like activity ( $P$ value of a paired two-sided $t$-test: 0.0007 and 0.0013 for PA28 $\gamma$ and PA28a $\beta$, respectively; Supplementary Fig. 2 and Supplementary Table 3). As expected, the PA28 proteasome-facing interfaces were highly protected in the complex compared to the regulators alone (Fig. 6a, b right, in blue; Supplementary Data 2, 12, 


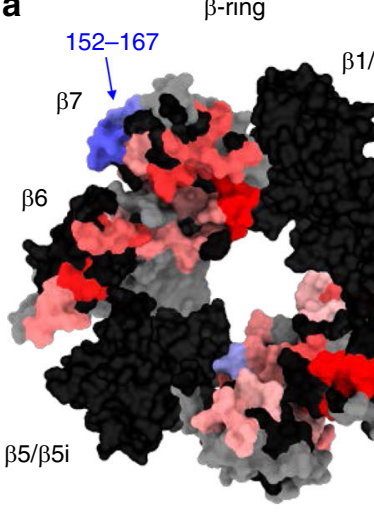

$\beta 4$

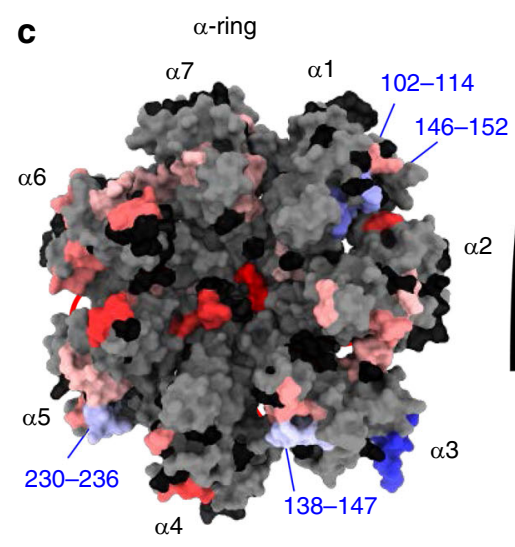

b

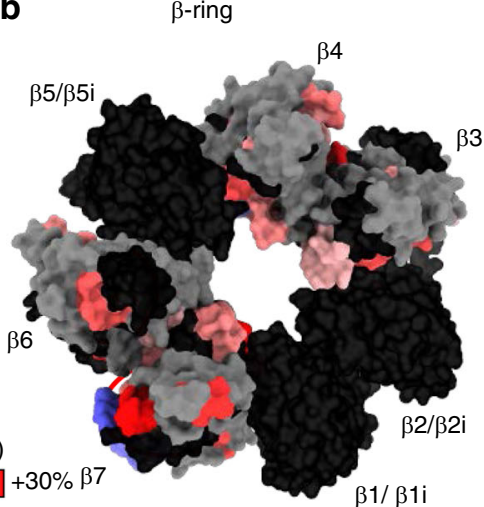

No RDU data

Not significantly regulated

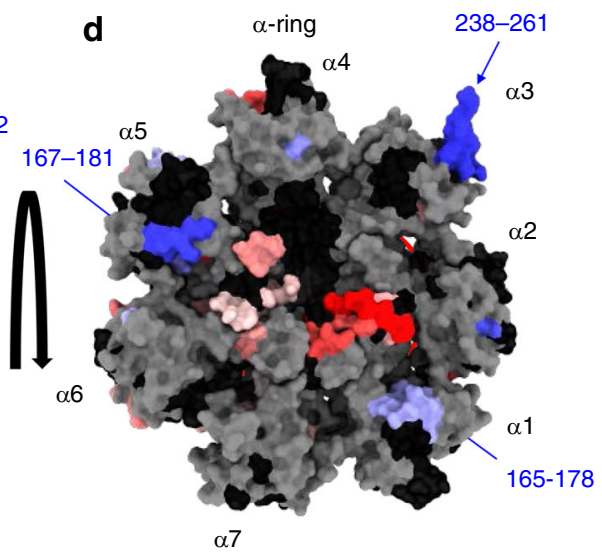

e

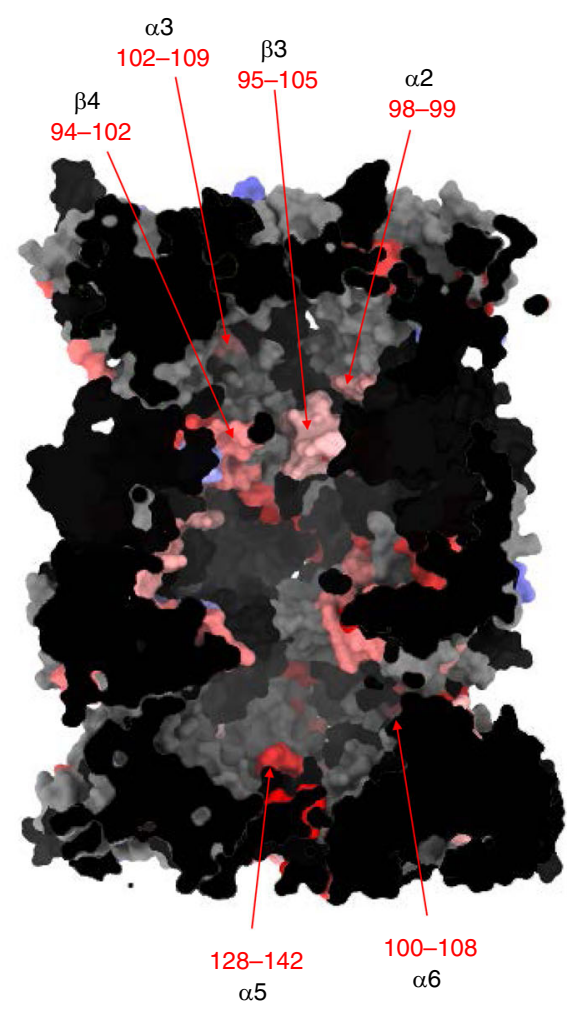

Fig. 4 Differential analysis of std20S and i20S. a-d Sum of differential relative uptakes between the i2OS and the $s t d 20 S\left(\Delta(R D U)=R D U_{i 20 S}-R U_{\text {std20S }}\right)$ : $\beta$-ring $/ \beta$-ring interface (a); $\beta$-ring facing the $\alpha$-ring (b); $\alpha$-ring facing the $\beta$-ring (c); solvent-facing $\alpha$-ring (d); inside view showing the antechambers and catalytic pockets (e). Color-coded regions were significantly more accessible/dynamic in the std20S (blue $=-30 \%$ ) or i20S (red $=+30 \%$ ) and regions of interest are indicated with arrows. Regions that are not covered in one or both conditions, or do not pass the statistical threshold are represented in black and grey, respectively (see Fig. 1 for more details). RDU relative deuterium uptake.

and 13). The activation loops, located at the $20 \mathrm{~S}$ binding interface, and known to interact with the N-ter of the 20S a subunits to open its central pore, were highly dynamic/accessible in both PA28 analyzed alone (Fig. 5d, e right, and Supplementary Data 11 and 12). Our data show that PA28 $\alpha \beta /$ std 20 S binding strongly protected the activation loops as well as their neighboring residues (136-147, 153-159, and 232-249 in PA28 $\alpha$; 123-135, 140-145 in PA28 $\beta$, Fig. 6a and Supplementary Data 2, 13, corrected $P$ values and RDU differences are available in Supplementary Data 9-10). The protection of PA28 $\gamma$ activation loop (region 146-152) upon std20S binding was also statistically significant but notably less pronounced (corrected $P$ value $=1.49 .10^{-8}$, sum of difference $=-20 \%$ ) (Fig. $6 \mathrm{~b}$ and Supplementary Data 9, 10).

Interestingly, other regions were less dynamic/accessible upon complex formation. The kinks of the first a-helix of PA28a (25-32; corrected $P$ value $=2.93 .10^{-15}$, sum of difference $=-61 \%$ ) and PA28 $\gamma$ (35-40; corrected $P$ value $=6.55 .10^{-13}$, sum of difference $=$ $-30 \%$ ) (Fig. 5a and Fig. 6a, b, red ovals) were strongly protected upon std20S binding. The PA28 $\beta$ N-ter (5-16) that faces PA28 $\alpha$ 's kink, was also significantly protected upon std20S interaction (corrected $P$ value $=9.19 \cdot 10^{-8}$, sum of difference $=-30 \%$ ) (Supplementary Data 2). We thus hypothesize that PA28a/PA28 $\beta$ interaction might be strengthened and/or structurally rearranged upon binding to the std20S. The N-ter (6-10) of PA28 $\gamma$ facing the kink was slightly but not significantly protected (corrected $P$ value $=$ $3.77 .10^{-3}$, sum of difference $=-6.4 \%$ ) (Fig. $6 \mathrm{~b}$ and Supplementary Data 2).
The opposite side of the heptamer was also partially protected upon std20S binding: the loops between helices 3 and 4 of PA28a (179-197), PA28ß (166-184), and PA28 $\gamma$ (203-208) (Fig. 6a, b) as well as the flexible loop (60-101) of PA28 $\gamma$ (Fig. 5b). These regions include the constriction sites at the top of PA28 $\alpha \beta$ (K187 and $\mathrm{K} 190$ in PA28a, K177 and K180 in PA28ß) that were significantly protected upon std20S binding (Fig. 6a and Supplementary Data 2). In PA28 $\gamma$, another constriction site, located underneath was protected upon std20S binding (R181, Fig. 6 b and Supplementary Data 2).

We then compared the impact of std20S vs. i20S binding on PA28 solvent accessibility using the statistical strategy presented in Fig. 1c (comparison referred to as PA28 + std20S vs. PA28+ i20S in Supplementary Data 2, 9, 10). Most of the reduction in accessibility/flexibility that was observed on the PA28 chains upon binding to the std20S were dampened upon interaction with the i20S (Fig. 6c, d and Supplementary Data 2).

A PA28-driven outer-to-inner allosteric change of the $20 \mathrm{~S}$ proteasome. Atomic force spectroscopy showed that the $20 \mathrm{~S}$ can alternate between open and closed states, possibly through allosteric regulation ${ }^{45,46}$. Our data indicate that PA28 $\alpha \beta$ and PA28 $\gamma$ do not present the same conformational rearrangements upon binding to the $20 \mathrm{~S}$, so they might allosterically affect the $20 \mathrm{~S}$ pore entrance and/or its catalytic sites in a regulator-specific outer-to-inner mechanism, as suggested recently ${ }^{25}$. 

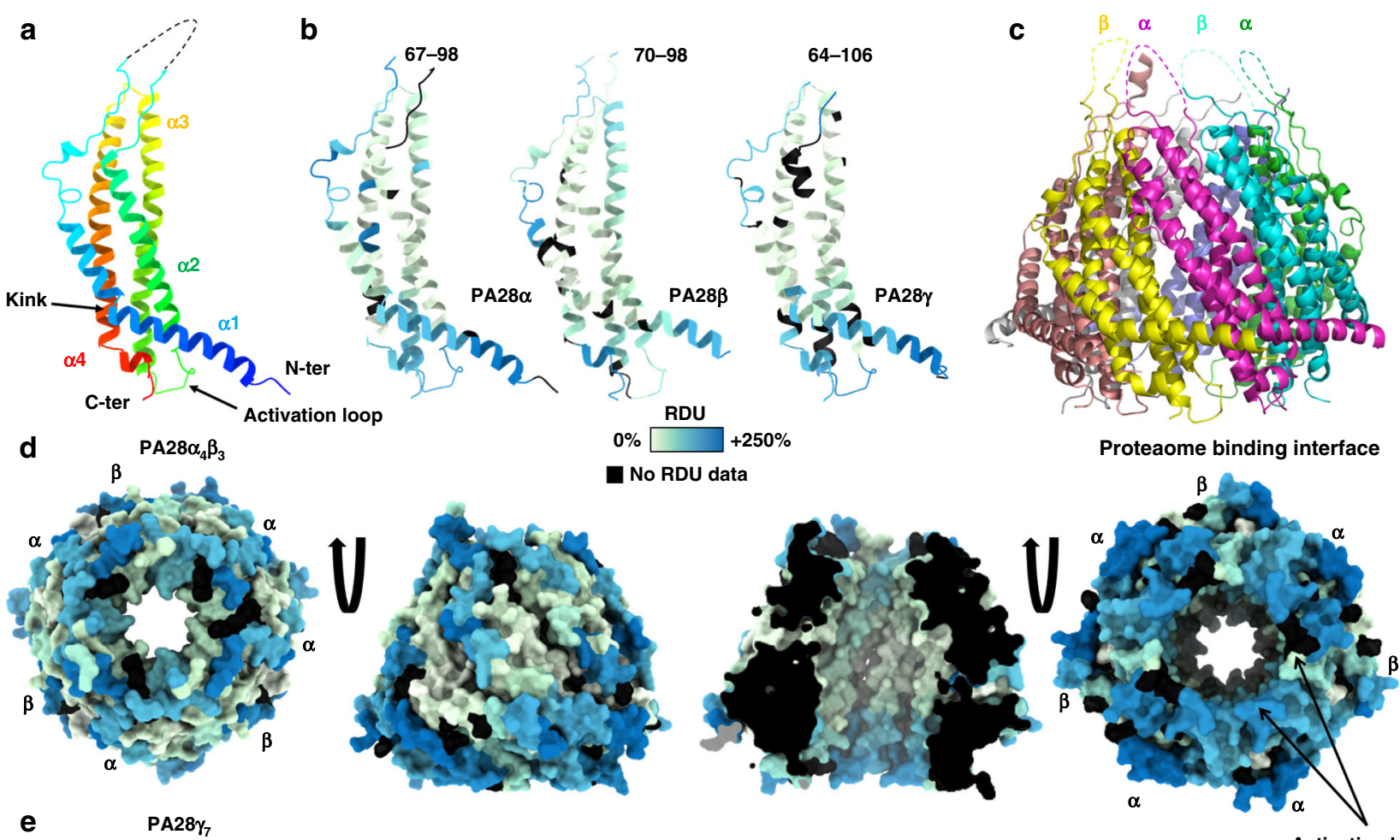

No RDU data
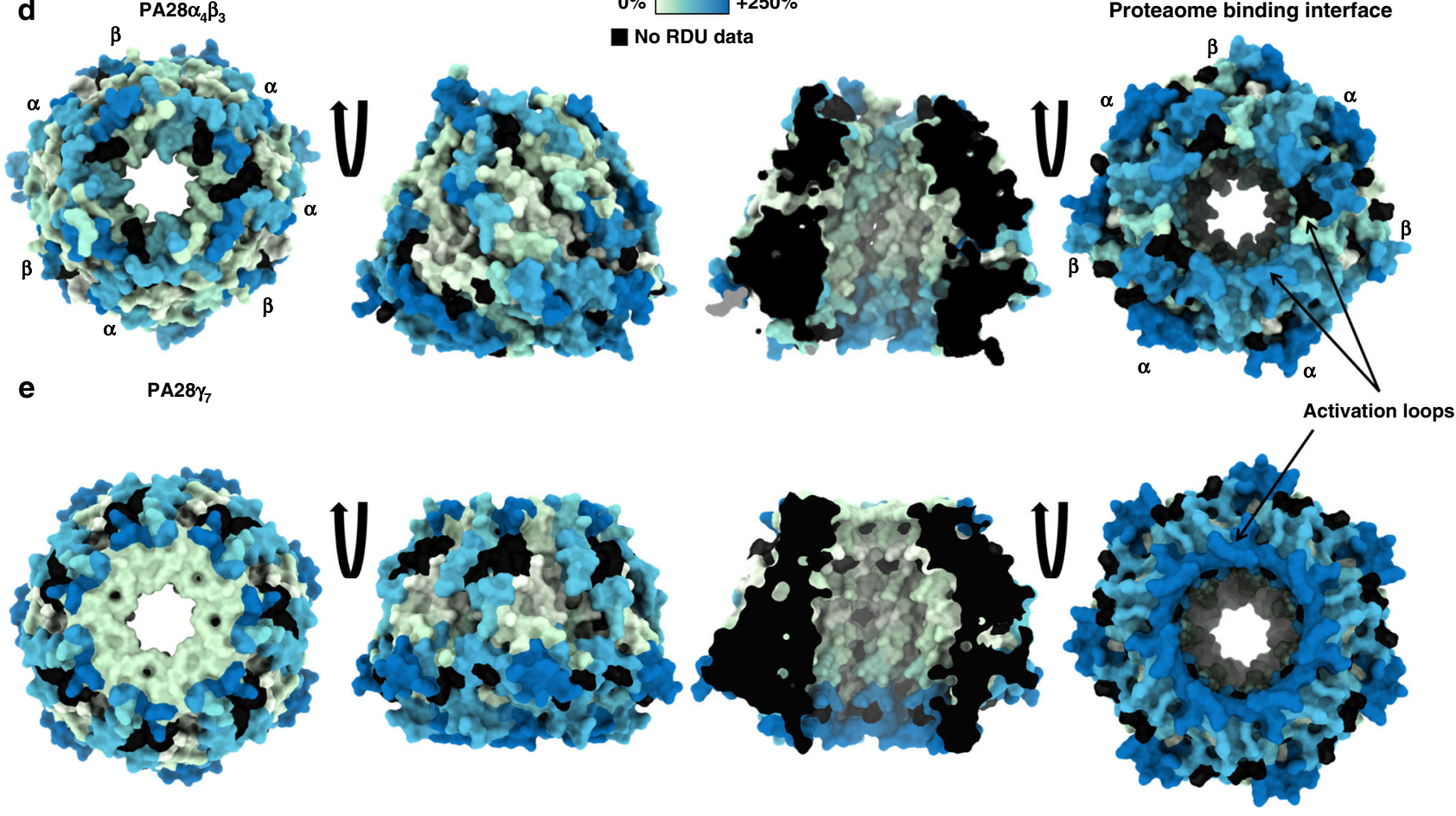

Fig. 5 PA28 regulators present flexible loops on both ends of the channel. $\mathbf{a}, \mathbf{b}$ Structure of monomeric PA2 $2 \alpha, \beta$, or $\gamma$ showing the 4 -helix-bundle colored based on amino-acid numbering (a) or the sum of hydrogen-deuterium relative uptake accumulated over 30 min in PA28 or PA28 $\alpha_{4} \beta_{3}$ in solution (b). The residues corresponding to the missing apical loops (dashed lines) are indicated in b. c Structure of the heptameric complex PA28 $\alpha_{4} \beta_{3}\left(P D B: 5 M \times 5^{33}\right.$ ). Only four apical loops out of seven are represented, for the sake of clarity. d, e Surface accessibility of the heptameric complexes PA28 $\alpha_{4} \beta_{3}$ (d PDB: $5 M \times 5$ ) and PA28 $\gamma_{7}$ (e homology model) viewed from the top (left), front and inside (middle), and bottom (right). Regions that were not detected are represented in black (described in Fig. 1b, c). RDU relative deuterium uptake.

We compared the std/i20S RDUs in presence or absence of PA28 $\alpha \beta$ or PA28 $\gamma$ (Fig. 7, Supplementary Data 3, 14, and 15). Strikingly, very few regions of the $\alpha$-ring in contact with the regulators were significantly protected in the std20S upon interaction with PA28 $\alpha \beta$ or PA28 $\gamma$, and none in the i20S. The same C-ter region of $\alpha 3$ (238-261) that was found to be more accessible in the std20S vs. i20S (corrected $P$ value $=1.55 \cdot 10^{-7}$, sum of difference $=-24 \%$ ) (Fig. $4 \mathrm{~d}$ ) was protected in the std20S upon interaction with PA28 $\alpha \beta$ (corrected $P$ value $=1.62 .10^{-5}$, sum of difference $=-28 \%$ ), and to a lesser extent with PA28 $\gamma$ (corrected $P$ value $=2.85 \cdot 10^{-4}$, sum of difference $\left.=-16 \%\right)($ Fig. $7 \mathrm{a}, \mathrm{f}$, red circle on $\alpha 3$ ). Other regions of the std20S that were protected when binding PA28 $\alpha \beta$ form two patches on the same solvent-exposed interface (Fig. 7a, red circles on $\alpha 4, \alpha 5$ ). These could be the main anchorage sites of PA28a $\beta$ at the surface of the std20S. The remainder of the std $20 \mathrm{~S}$ solvent-exposed surface was globally more dynamic upon PA28a $\beta$ binding, especially near the pore entrance (N-ter of $\alpha 1 / 2 / 3 / 4 / 5 / 7$ ), which can be interpreted as an opening of the pore (Fig. 7a, large red circle). Another external patch at the $\alpha 1 /$ $\alpha 2$ interface was also more dynamic upon binding of PA28a $\beta$ (Fig. 7a, red circle on $\alpha 1, \alpha 2$ ).

On the $\beta$-facing $\alpha$-ring, PA28 $\alpha \beta$ binding mainly destabilized $\alpha 2$ (136-145: corrected $P$ value $=2.48 .10^{-5}$, sum of difference $=$ $10 \%)$ and the $\alpha 5$ bulge (102-107: corrected $P$ value $=1.56 .10^{-5}$, sum of difference $=19 \%$ ) (Fig. $7 b$, red circles on $\alpha 2$, $\alpha 5$ ). Interestingly, this bulge faces the 109-135 $\beta 6$ stretch encompassing 4 peptides more deuterated upon PA28a $\beta$ binding and is in close vicinity to the $\beta 5$ interface, which is also more dynamic (Fig. $7 \mathrm{c}$, red circles on $\beta 5, \beta 6$, corrected $P$ values and RDU differences are available in Supplementary Data 9-10). Although not close enough to make hydrogen bonds, a 2 (136-145) (Fig. 7b, red circle on $\alpha 2$ ) faces the $\beta 2 / \beta 3$ interface that was more flexible/ accessible upon PA28a $\beta$ binding, especially in the 186-202 C-ter region of $\beta 2$ extending towards $\beta 3$ (Fig. $7 \mathrm{~d}$, circle on $\beta 2$ ). The only peptides of the $\beta$-ring that were less dynamic upon PA28a $\beta$ binding are located on $\beta 1$ (Fig. $7 c$, red circle on $\beta 1$ ). 
a $\mathrm{PA28} \alpha_{4} \beta_{3}+\mathbf{s t d 2 0 S}$
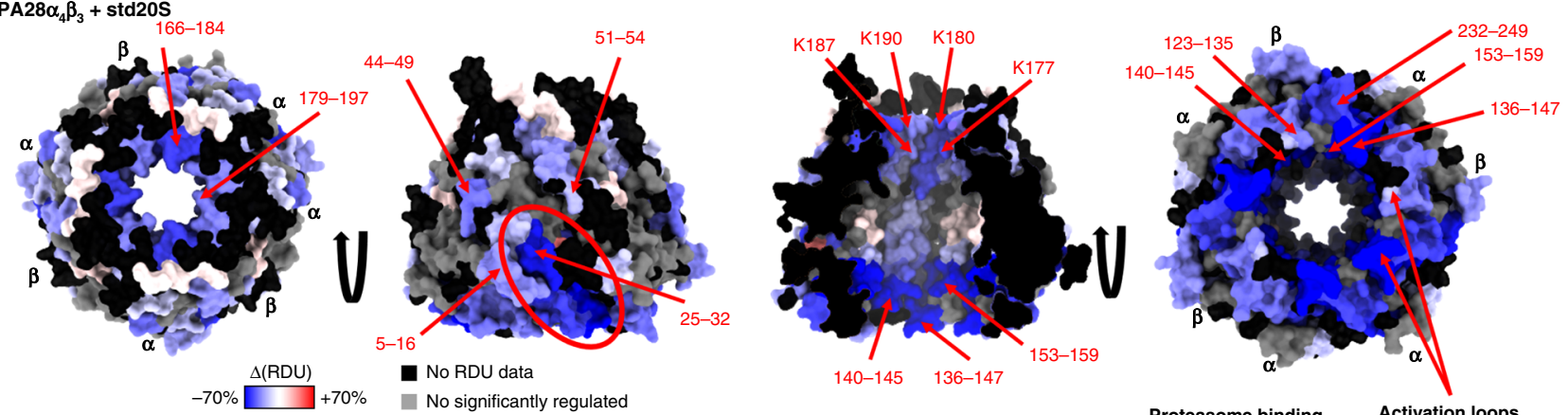

b PA28 $\gamma_{7}+\operatorname{std} 20 \mathrm{~S}$
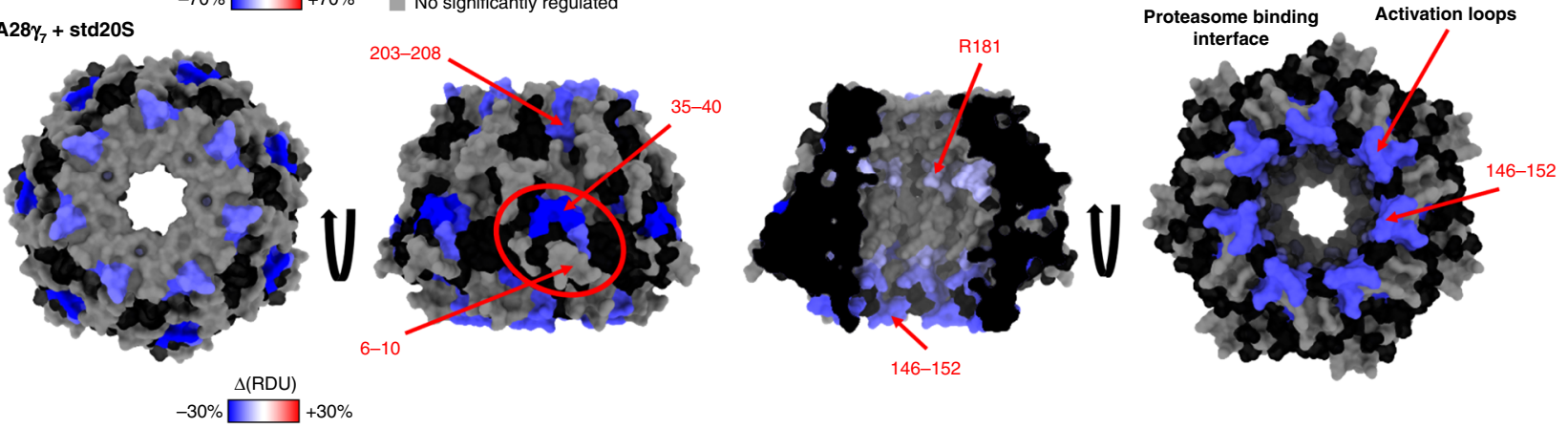

C PA28 $\alpha_{4} \beta_{3}+i 20 S$
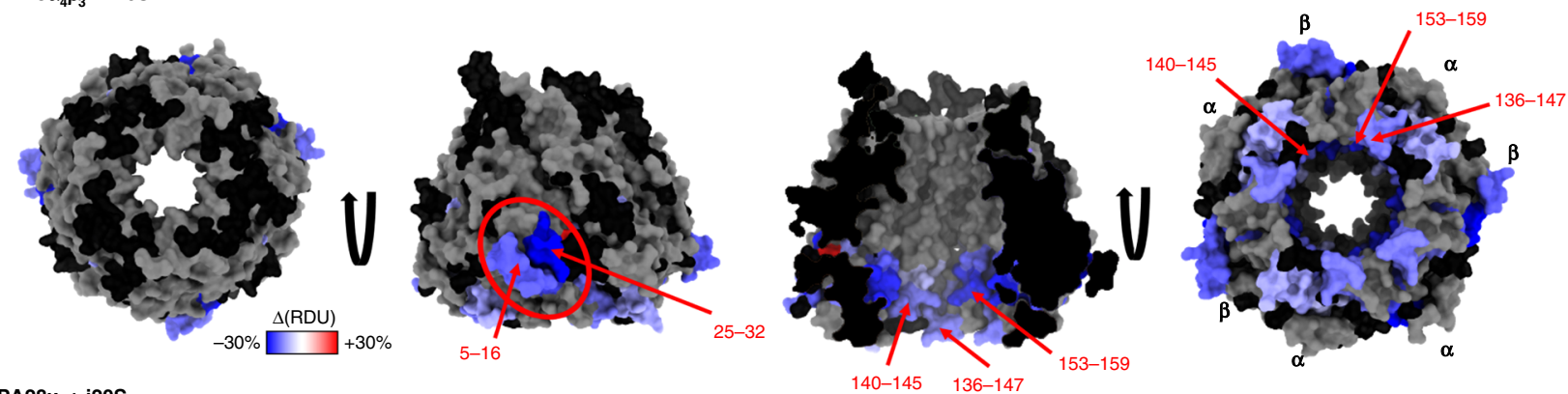

d $\mathrm{PA}_{28} \gamma_{7}+\mathrm{i} 20 \mathrm{~S}$
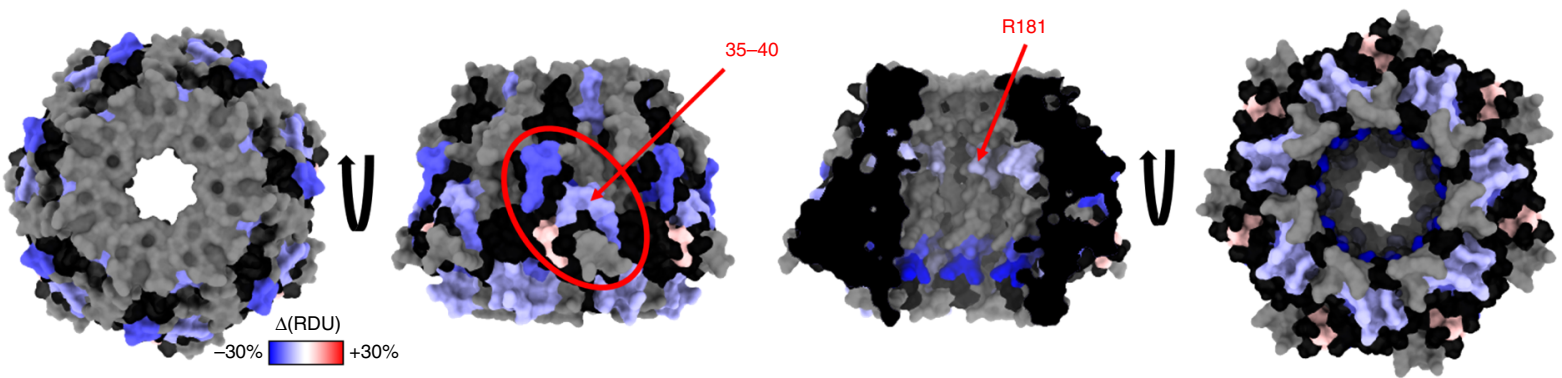

Fig. 6 Activation of PA28 $\alpha \boldsymbol{\beta}$ and PA28 $\gamma$ by the 20 S involves a rearrangement of the $\mathbf{N}$-ter kink and an allosteric change. The sum of differential RDUs accumulated from 0.5 to 30 min deuteration of the PA28 regulators alone or in presence of the $20 \mathrm{~S}$ proteasome $\left(\triangle(R D U)=R D U_{P A 28 / 20 S}-R D U_{P A 28}\right)$ is color coded from blue (less accessible in the complex) to red (more accessible in the complex) and ranges from $-70 \% /+70 \%$ for PA28 $\alpha \beta+$ std20S (a) and $-30 \% /+30 \%$ for PA $28 \gamma+\operatorname{std} 20$ S (b) PA2 $2 \alpha \beta+\mathrm{i} 20 \mathrm{~S}$ (c) and PA28 $\gamma+\mathrm{i} 20 \mathrm{~S}$ (d). The heptameric complexes PA28 $\alpha_{4} \beta_{3}(\mathbf{a}$ and $\mathbf{c}$ PDB: $5 \mathrm{MX} 5$ ) and PA28 $\gamma_{7}$ (b and $\mathbf{d}$ homology model) are viewed from the top (left), front and inside (middle), and bottom (right). Residues of PA28 that present a significant decrease of deuteration upon binding of the $20 \mathrm{~S}$ proteasome are located around the N-ter kink (red ovals), the activation loop and 20S interface and the entry of the channel (left). Residues in gray were not considered significantly different according to the statistical thresholds presented in Fig. 1c. Residues with no RDU information in one or both conditions are in black. $\Delta(R D U)$ sum of differences of relative deuterium uptake.

We compared the effect of PA28a $\beta$ binding to the std20S (Fig. $7 \mathrm{a}-\mathrm{e}$ ) with the other conditions (Fig. 7f-t). Although the destabilization of the $\alpha$-ring $\mathrm{N}$-ter by PA28 $\alpha \beta$ was almost complete in the std20S (except $\alpha 6$ ) (Fig. 7a, large red circle), it was restricted to $\alpha 2 / 3 / 7$ in the i20S (Fig. $7 k$, large red circle), suggesting a partial opening of the pore. Binding of PA28 $\gamma$ did not affect any of the std20S a subunit N-ter (Fig. 7f) and only the i20S $\alpha 2$ and $\alpha 7 \mathrm{~N}$-ter (Fig. 7p, large red circle), indicating less PA28 $\gamma$-driven opening of the std20S than the i20S pore.
Overall, PA28 binding to the std/i20S increased their RDU, especially at the pore entrance, at the $\alpha 1 / \alpha 2$ interface (Fig. $7 \mathrm{a}, \mathrm{k}, \mathrm{p}$, red circles on $\alpha 1, \alpha 2)$, and the $\beta 2 / \beta 2 \mathrm{i}$ C-ter arm extending towards $\beta 3$ (Fig. $7 \mathrm{~d}, \mathrm{e}, \mathrm{n}, \mathrm{o}, \mathrm{s}, \mathrm{t}$ ). Despite these similarities, our data show subtle proteasome- as well as regulator-specific differences in the allosteric motion triggered upon complex formation, as suggested recently ${ }^{25}$. The std20S a3 C-ter was protected upon binding of PA28a $\beta$ and PA28 $\gamma$ (Fig. 7a, f). This seemed to induce the PA28 $\gamma$-specific destabilization of $\alpha 2$ 


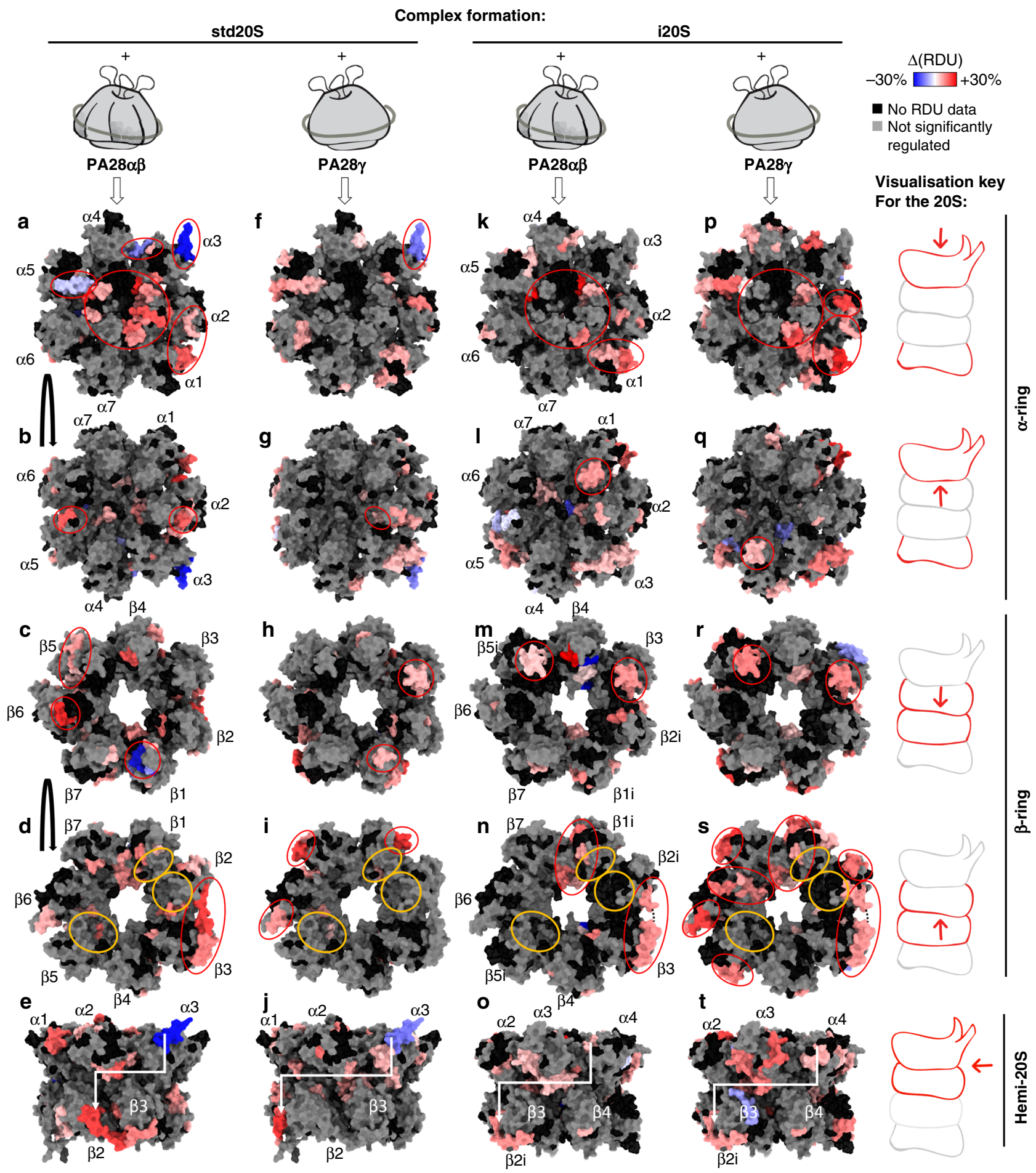

Fig. 7 Catalytic-subunit- and regulator-specificity of the outer to inner allosteric changes. Differential relative uptakes accumulated over 30 min deuteration of the $20 S$ proteasomes with and without the PA28 regulators $\left(\triangle(R D U)=R D U_{20 S / P A 28}-R_{2} U_{20 S}\right.$ ). The regions that were statistically protected or more dynamic upon regulator binding are represented in blue and red, respectively, for the std20S (PDB: 5LE5) and PA28 $\alpha \beta$ (PDB: 5MX5) (a$\mathbf{e})$, the std20S and PA28 $\gamma(\mathbf{f}-\mathbf{j})$, the i2OS (PDB: 6E5B) and PA28 $\alpha \beta(\mathbf{k}-\mathbf{0})$, and the i20S and PA28 $\gamma(\mathbf{p}-\mathbf{t})$. Active sites at the $\beta$-ring/ $\beta$-ring interface are circled in orange. The side views of the hemi-proteasome $\mathbf{j} / \mathbf{o} / \mathbf{t} / \mathbf{e}$ show the $\alpha$-ring and $\beta$-ring interface. Residues in gray were not considered significantly different according to the statistical thresholds presented in Fig. 1c. Residues with no RDU information in one or both conditions are in black. $\Delta$ (RDU) sum of differences of relative deuterium uptake. Regions discussed in the text are circled in red. Missing residues from the $\mathrm{C}$-terminal part of $\beta 2 i$ are represented as a black dotted line in panels $\mathbf{n}$ and $\mathbf{s}$. The visualization key on the right indicates the identity and orientation of the structures with the same schematics of the $20 \mathrm{~S}$ as in Fig. 8. 
(101-119: corrected $P$ value $=4.03 \cdot 10^{-5}$, sum of difference $=$ $13 \%$, Fig. $7 \mathrm{~g}$, red circle on $\alpha 2), \beta 3$ (74-86: corrected $P$ value $=$ $1.19 .10^{-6}$, sum of difference $\left.=14 \%\right)$, and $\beta 1(64-70$ : corrected $P$ value $=8.72 .10^{-4}$, sum of difference $\left.=10 \%\right)$ bulges $($ Fig. $7 \mathrm{~h}$, red circles on $\beta 1, \beta 3)$, down to the outer $\beta$-ring/ $\beta$-ring interface of $\beta 1$ (178-205), $\beta 6$ (151-159: corrected $P$ value $=1.40 .10^{-7}$, sum of difference $=13 \%)$ and $\beta 7(152-167)$ (Fig. $7 \mathrm{i}$, red circles on $\beta 1, \beta 6$, $\beta 7$; see Supplementary Data 8 for the corrected $P$ values and RDU differences corresponding to the protein portions covered with multiple peptides). According to our statistical thresholds, the $\alpha 3$ C-ter protection upon PA28 binding was std20S-specific (Fig. 7k, $\mathrm{p})$, but the remodeling of the $\alpha$-ring occurred in both $20 \mathrm{~S}$ (although differently) and was propagated to the $\beta$-ring of the i20S via $\alpha 1 / \beta 3 / \beta 5$ i and $\alpha 4 / \beta 3 / \beta 5$ i bulges for PA28 $\alpha \beta$ (Fig. $71, m$, red circles on $\alpha 1 / \beta 3 / \beta 5 i$ ) and PA $28 \gamma$, respectively (Fig. $7 \mathrm{q}, \mathrm{r}$, red circles on $\alpha 4 / \beta 3 / \beta 5 i)$. It resulted in remodeling of the $\beta$-ring $/ \beta$ ring interface eventually affecting $\beta 1 \mathrm{i} / 2 \mathrm{i} / 7$ or $\beta 1 \mathrm{i} / 2 \mathrm{i} / 5 \mathrm{i} / 6 / 7$ with PA28 $\alpha \beta$ (Fig. $7 \mathrm{n}$, red circles on $\beta 1 \mathrm{i} / 2 \mathrm{i} / 7$ ) and PA28 $\gamma$ (Fig. $7 \mathrm{~s}$, red circles on $\beta 1 \mathrm{i} / 2 \mathrm{i} / 5 \mathrm{i} / 6 / 7)$, respectively.

The limited sequence coverage around the catalytic sites prevented us from drawing exhaustive conclusions on the differential impact of regulator/20S complex formation on the proteasome active sites. However, $\beta 1(10-25)$ (corrected $P$ value $=$ 9.49.10 ${ }^{-9}$, sum of difference $\left.=22 \%\right), \beta 2(16-42)$ (corrected $P$ value $=9.54 .10^{-5}$, sum of difference $\left.=5.2 \%\right)$ and $\beta 5(29-36)$ (corrected $P$ value $=8.84 .10^{-3}$, sum of difference $=8 \%$ ) active sites were partially but significantly more dynamic/accessible upon PA28 $\alpha \beta$ binding (Fig. 7d, orange circles and Supplementary Data 3 and 14), in-line with an increased proteolytic activity. Binding of PA28a $\beta$ also increased the solvent accessibility of $\beta 1 \mathrm{i}$ active site (10-17) (corrected $P$ value $=3.89 .10^{-3}$, sum of difference $=14 \%$ ) (Supplementary Data 3 and 14), which is in good agreement with previous work showing that the caspase-like activity of the $i 20 \mathrm{~S}$ is enhanced to a higher extent by PA28a $\beta$ than PA28 $\gamma^{53}$. PA28 $\gamma$ was reported to have little or no effect on the $\mathrm{i}_{20 \mathrm{~S}^{44}}$, whereas it is known to increase all three std20S activities ${ }^{54}$. Our data do not indicate any major change in the std20S active site solvent accessibility upon interaction with the regulator (Fig. 7i). However, it revealed a significant decrease of $\beta 2 \mathrm{i} \mathrm{N}$-ter $(2-6)$ (corrected $P$ value $=3.80 .10^{-3}$, sum of difference $=-13 \%$ ) solvent accessibility upon PA28 $\gamma$ interaction (Supplementary Data 3). Thus, the catalytic activation observed upon PA28 $\gamma /$ std20S binding may be driven by changes occurring further away from the active sites.

\section{Discussion}

This structural dataset on the human i20S compared to the std20S alone or bound to both PA28 regulators rationalizes from a mechanistic point of view previous observations that replacement $^{49}$, modification ${ }^{34}$ or ligand binding ${ }^{49,55}$ to the catalytic subunits can allosterically modify the $20 \mathrm{~S}$ core particle structure and alter its binding to potential PIPs. A main advantage of HDX-MS is that it provides information on very flexible loops, unlike X-ray crystallography or cryo-EM. Also, it is not theoretically limited by the complex size since the proteins are digested into peptides after deuteration: it can be applied to very small proteins (unlike cryo-EM) or very large complexes (unlike NMR). Large oligomeric complexes containing a reduced number of different monomers (limited number of chemical shifts) have already been analyzed by NMR, such as the prokaryotic $20 \mathrm{~S}$ from T. acidophilum constituted of 14 identical $\alpha$ and 14 identical $\beta$ subunits $^{34}$. With the recent exception of the DNA-PKc analysis ${ }^{56}$ (469 $\mathrm{kDa}$ monomer), HDX-MS is usually performed either on rather small and simple systems ${ }^{39}$ or on homo-oligomeric complexes $^{38,57}$, due to some technical limitations in protein digestion, peptide separation and data analysis. In comparison, our study encompasses 16 different $\sim 25 \mathrm{kDa}$ monomers. Despite this analytical challenge, we obtained an average sequence coverage per subunit of $89 \%$ for the 20 S alone and $81 \%$ when in complex with PA28 regulators. It is important to note that, except for a few specific cases ${ }^{58,59}$, HDX-MS does not inform on the presence of multiple conformational states in solution since it is based on the measured average of their RDUs. Other approaches such as cryo-EM-based classification would be needed to better characterise intermediate or minor states. Nevertheless, comparing the same sample (like std20S) in presence or absence of regulator and performing thorough statistical analysis identified a wealth of information on the proteasome dynamics, binding interfaces and allosteric changes upon incorporation of the immuno-subunits or activation by PA28 regulators (Fig. 8).

These conformational maps revealed the subtle rearrangement of the std/i20S a3 C-ter. More precisely, the residues 238-261 were less dynamic/accessible upon incorporation of the immunocatalytic subunits (in blue Fig. 8a). The principal component analysis of the 20S high-resolution structures available in the Protein Data Bank (PDB) in 2014 (46 structures from yeast, 4 murine and 1 bovine) revealed that the 220-230 stretch of the mouse std20S and i20S $\alpha 3$ clustered with the yeast apo and peptide-bound forms, respectively ${ }^{49}$. We believe that the allosteric change occurring on $\alpha 3 \mathrm{C}$-ter upon ligand binding to $\beta 5$ in the yeast $20 \mathrm{~S}$ could be similar to the change in solvent accessibility/ dynamics observed between the std20S and the i20S conformational maps. The highly charged C-ter of $\alpha 3$ points towards the outer surface of the a-ring and has also been suggested to be the Insulin-Degrading-Enzyme binding site ${ }^{60}$. It could be involved in the binding of PA28 $\beta / \gamma$ to the std20S, or in its subsequent activation. Interestingly, the very end of $\alpha 3 \mathrm{C}$-ter is absent from most human $20 \mathrm{~S}$ proteasome structures, which confirms its high flexibility but also impedes any modeling of 20S/PA28 interaction. Unfortunately, this protruding C-ter a helix is not present in the cryo-EM structure of the $P f 20 \mathrm{~S}$ bound to its PA28 regulator, since it is nine residues shorter than its human counterpart ${ }^{36}$. It could be characteristic of more evolved $20 \mathrm{~S}$ proteasomes and tune their binding with different regulators.

As commented before, the differential analysis of the catalytic subunits is impossible due to their sequence differences (the peptides resulting from the pepsin digestion are different). The comparison of their deuteration profiles was further hindered by the limited sequence coverage of the i20S. This was partly due to the presence of residual traces of std20S present in this sample 61 . Nevertheless, it was possible to compare the RDUs of the noncatalytic subunits of the two core particles. The central pore of the 20S was more flexible in the i20S vs. std20S (Fig. 4d and in red Fig. $8 \mathrm{a}$ ), as was the $\beta$-facing interface of the $\alpha$-ring, especially $\alpha 5$ that directly faces the $\beta 5$ (std20S) or $\beta 5 \mathrm{i}$ (i20S) catalytic subunit (Fig. 4c). We propose that the conformational differences observed between the standard and immuno- $\alpha 3 \mathrm{C}$-ter (red arrows Fig. 8a) could be transduced from the inner $\beta$-ring to the outer $\alpha$ ring via $a 5$.

Interestingly, upon binding of any regulator to the 20S, we observed a similar destabilization of the $\alpha 5$ region that faces $\beta 5 / 5 i$, suggesting that the exact opposite mechanism (from the outer $\alpha$ ring to the inner $\beta$-ring) can also take place upon 20S/PA28 interaction, as suggested earlier ${ }^{34}$.

Overall, our data indicate a general mechanism of PA28 activation by the $20 \mathrm{~S}$ core proteasome. More precisely, the interaction of PA28 activation loops and C-ter with the $20 \mathrm{~S}$ seems to trigger a long-range allosteric change at the top of the regulator (loops between helices $1 / 2$ and/or 3/4) via a rearrangement of the N-ter kink of PA28a and PA28 $\gamma$ (Fig. 8b). Although present in the three PA28 subtypes, the activation loop protection was stronger in PA28 $\alpha$ and PA28 $\gamma$ vs. PA28 $\beta$; most probably 
a Inner-to-outer conformational changes between std20S and i20S

Pore entrance

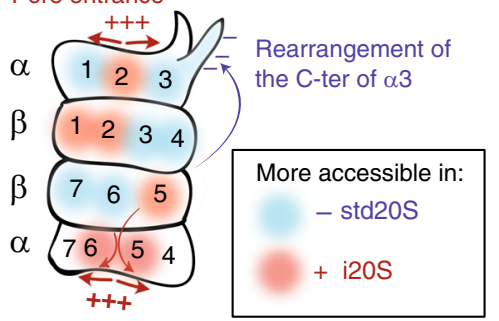

b Solvent accessibility/flexibility of PA28 $\alpha \beta$ and PA28 $\gamma$ alone

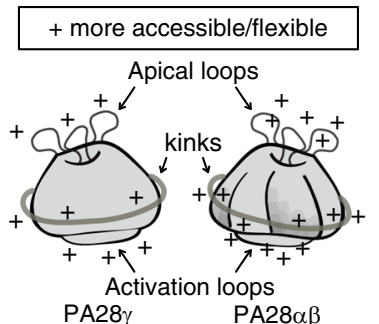

Proteins

alone

C Outer-to-inner conformational changes upon regulator binding

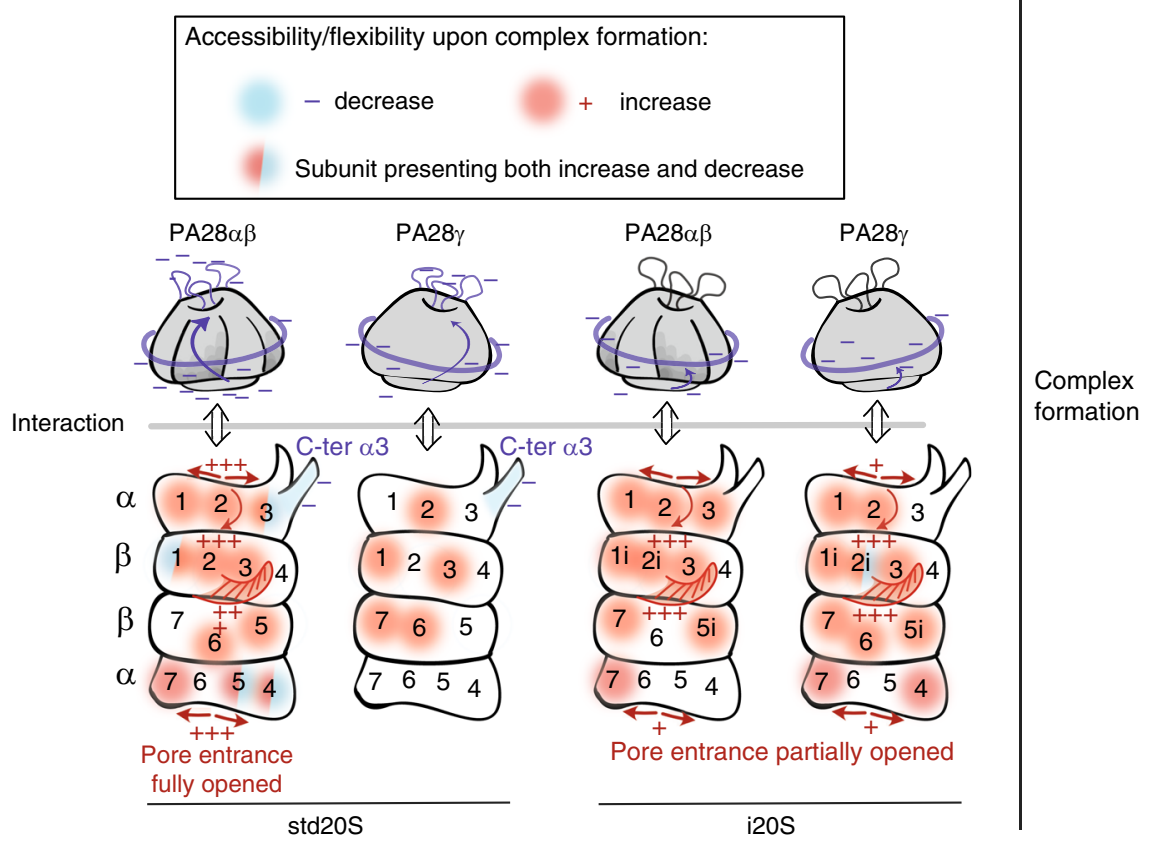

Fig. 8 Schematic representation of our major finding. a Comparison of std2OS vs. i20S deuteration profiles confirms an inner-to-outer allosteric change: the differences between the std20S- and i20S-specific subunits $\beta 1 / 1 \mathrm{i}, \beta 2 / 2 \mathrm{i}$ and $\beta 5 / 5 \mathrm{i}$ located inside the complex trigger conformational changes at the pore entrance (aperture in the i20S: +++ ) and external surface of the proteasome, including the C-ter of $\alpha 3$ (inner-to-outer change represented as an arrow). The subunits that are more accessible/flexible in the std20S and the $\mathrm{i} 20 \mathrm{~S}$ are presented in blue and red, respectively. b PA28 solvent accessibilities analysed alone indicate similarities in their dynamic regions: their apical loops, kinks and activation loops were more deuterated (indicated with+). c 20Sand PA28-specific reorganizations occurring upon complex formation. This schematics presents the results of the comparison of HDX-MS data from the PA28 and std/i20S alone vs. in complex. The subunits in red and blue were globally more and less deuterated in the complex than in the protein alone, respectively. More localised changes are indicated by half-circles. Opening of the 205 pore entrance is indicated with + and +++ (for minor and more pronounced increase of deuteration) as well as the reorganisation of the $C$-ter of $\beta 2$ and $\beta 2 i$ upon complex formation. - indicates the area of the proteins that were protected upon binding (apical loops, kinks and activation loops of the regulators; C-ter of $\alpha 3$ in the std20S).

explaining why "the affinity of the proteasome towards the PA28 $\beta$ complex is about two orders of magnitude higher than towards the homomeric PA28 $\alpha$ and PA28 $\beta$ complexes" 62 . Indeed, several studies showed that both PA28 $\alpha$ and PA28 $\beta$ were required for an optimum activation of the $20 \mathrm{~S}$ by PA28a $\beta^{32,33}$.

We also provide crucial information on the loops located between helices $1 / 2$, missing in every PA28 high-resolution structure or model. Since these are located at the entrance/exit of the activator, any change in their flexibility could modify the nature of both substrates and/or products of the proteasome complex. Indeed, it has been shown in PA28a to constrain substrates within the catalytic chamber of the core particle, thereby generating shorter peptides ${ }^{63}$. We show that these flexible loops, together with PA28 constriction sites (conserved lysine between alpha helices 3 and 4) are allosterically stabilized upon binding of the std20S but not the i20S (Fig. 8c). This could explain how the binding of different PA28 to different 20S subtypes alters the length/nature of the generated peptides. Our data also suggest that PA28 might modify the 20 S products by inducing conformational changes in the proteasomal active sites. Indeed, our results show distinctive changes in dynamics detected down to the $\beta$-ring and around the catalytic site and/or substrate pockets upon regulator binding (Fig. 8c).

We observed a stronger protection of PA28 $\alpha \beta$ than PA28 $\gamma$ upon std20S binding (Fig. 6 and Fig. 8c). Globally, both PA28 were more protected upon interaction with the std20S than with the i20S (Fig. 8c and Supplementary Data 2 and 13), suggesting a stronger interaction of the regulators with the std20S than the i20S (Supplementary Data 2). This was mirrored by regions on the i20S $\alpha$-ring that were less protected than in the std20S upon incubation with both PA28, whereas previous studies showed that PA28 $\alpha \beta$ interaction was stronger with the i20S than with the $\operatorname{std} 20 S^{64}$ or equivalent ${ }^{21}$. In this context, we think that protection to deuteration cannot be always correlated to relative binding 
affinities. The very limited decrease of $\mathrm{RDU}$ on the $\alpha$-ring interface interacting with PA28 regulators can be explained by the resulting gate opening (RDU increase) known to induce its increased proteolytic activity. Gate opening and binding interface protection are expected to have opposite impacts on RDU, so their combination in the same protein region would result in no significant changes of deuteration rates. We showed that both PA28 regulators activate the chymotrypsin-like activity of the std20S to a similar extent in our in vitro assay (Supplementary Fig. 2). However, the N-ter portions of the std20S a subunits (entrance of the channel) were significantly more deuterated when interacting with PA28a $\beta$ whereas this increase was not significant with PA28y (Fig. 7a, f and Supplementary Data 3), suggesting that their allosteric mechanisms of activation are different. This could alternatively be explained by a shorter dwelltime at the surface of the std20S or by differences in binding stoichiometry. More experiments are needed to fully understand these allosteric mechanisms of activation.

Although not similarly distributed, a global core proteasome destabilization upon complex formation was found in all four conditions tested (Fig. 7), so we focused on the comparison of the most affected regions. We observed that each pair of PA28/20S complex underwent long-range conformational rearrangements in specific regions, as suggested recently $y^{25}$. Different sets of $\alpha \mathrm{N}$ ter were destabilized depending on the PA28/20S pair (in red Fig. 8c), suggesting a gradual interaction-specific opening of the central pore, in-line with a recent cryo-EM structure that showed a partial opening of the bovine i20S gate in complex with human PA28 $\alpha \beta^{65}$. The $\beta 2 \mathrm{C}$-ter extending towards $\beta 3$ was destabilized in all the conditions, except std20S/PA28 $\gamma$. On the contrary, the region just before the a3 C-ter (217-236) was protected upon PA28 binding in all cases, except std20S/PA28a $\beta$. Interestingly, this stretch is close to $\beta 374-86$, which was also protected in all conditions tested except std20S/PA28 $\beta$. These observations could provide a basis to understand how regulator binding propagates from the $\alpha$ - to the $\beta$-ring depending on the activator/ 20S pair.

Altogether, these data provide a unique resource informing on the allosteric activation of the $20 \mathrm{~S}$ proteasome complexes that should soon be completed by upcoming structural and functional studies. We think that HDX-MS could be applied to characterize, not only the binding sites of many new proteasome inhibitors, but also to identify their potential allosteric effects on the $20 \mathrm{~S}$ core complex. The same is true for the many PIPs that regulate proteasome function and are still poorly characterized from a structural point of view ${ }^{66}$. More broadly, this work demonstrates the ability of HDX-MS to investigate dynamic events on megadalton assemblies including ribosomal particles, inflammasomes, and nucleosomes, to name a few.

\section{Methods}

Reagents. Unless stated otherwise, all reagents were purchased from Sigma-Aldrich. The std20S (BML-PW8720-0050), i20S (BML-PW9645-0050), PA28 $\gamma$ (BML-PW9875-0100), and PA28 $\alpha \beta$ (BML-PW9420-0025) were purchased from Enzo Life Science. Deuterium oxide (D215H) and sodium deuteroxide (D076Y) solutions were from Euriso-top and deuterium chloride (543047-50 G) was from Sigma-Aldrich. The fluorogenic peptide substrate (4011369.0025) for proteasome activity measurement was from Bachem.

Proteasome activity test. The assay was performed in black 96-well plates (Fluotrac 200-GREINER BIO-ONE). $5 \mu \mathrm{L}$ of each sample (corresponding to $50 \mathrm{ng}$ of $20 \mathrm{~S}$ and/or $28 \mathrm{ng}$ of PA28) were added in three independent experiments to 45 $\mu \mathrm{L}$ of Tris- $\mathrm{HCl} 100 \mathrm{mM} \mathrm{pH} 8$ and $50 \mu \mathrm{L}$ of Suc-LLVY-AMC (for chymotrypsin-like activity) substrate in $200 \mathrm{mM}$ Tris- $\mathrm{HCl}, \mathrm{pH} 8$ at a final concentration of $400 \mu \mathrm{M}$ / well. The kinetic assays were performed at $37^{\circ} \mathrm{C}$ in a FLX-800 spectrofluorimeter (BIOTEK, Winooski, VT, U.S.A.) over $90 \mathrm{~min}$ with one reading every $5 \mathrm{~min}$, at 360 $\mathrm{nm}$ for excitation and $460 \mathrm{~nm}$ for emission. Each proteasome specific activity was obtained by dividing the activity by the proteasome quantity ( $50 \mathrm{ng}$ ). A two-sided $t$ test was used between the std20S alone and in presence of PA28.

Development of a HDX-MS pipeline dedicated to the comparative analysis of $20 S$ proteasome/regulator complexes. In a classical HDX-MS workflow, the proteins of interest are digested online and the generated peptides are separated on a reverse phase column before MS analysis to monitor deuterium incorporation rates. The main challenges encountered when analysing heterogeneous complexes such as the proteasome by HDX-MS are threefold. First, the high number of peptides generated after digestion entailed specific optimization of their chromatographic separation. Second, we optimized the quenching step and injection parameters to reduce dead volumes, as well as the acquisition method, in order to handle samples at low concentration $(<1 \mu \mathrm{M})$. Finally, we developed a computational pipeline dedicated to the multidimensional analysis of HDX-MS analysis of large complexes. The resulting data were mapped to available 3D structures using our recently developed open-source web application HDXViewer v.1.267

\section{Automated Hydrogen-Deuterium eXchange coupled to Mass Spectrometry} (HDX-MS). HDX-MS experiments were performed on a Synapt-G2Si (Waters Scientific, Manchester, UK) coupled to a Twin HTS PAL dispensing and labelling robot (LEAP Technologies, Carborro, NC, USA) via a NanoAcquity system with HDX technology (Waters, Manchester, UK). Data were collected with MassLynX v.4.1 from Waters Scientific (Manchester, UK) and the robot was controlled via HDx Director v.1.0.3.9 (LEAP Technologies, Carborro, NC, USA). Each step was optimized to minimize sample loss and work with such a heterogeneous and diluted sample:

Method in HDxDirector: The method recommended by Waters (HDX System Suitability Test) injects only $25 \%$ of the sample that is aspirated from the protein vial. In order to reduce sample loss, we (1) used a sample loop of $100 \mu \mathrm{l}$ instead of $50 \mu \mathrm{l},(2)$ carefully reduced all the dead volumes, and (3) used $500 \mathrm{mM}$ glycine $\mathrm{pH}$ 2.3 instead of $50 \mathrm{mM} \mathrm{K}_{2} \mathrm{HPO}_{4}, 50 \mathrm{mM} \mathrm{KH}_{2} \mathrm{PO}_{4}, \mathrm{pH} 2.3$ in order to optimize the ratio of quenching volume $(10 \%$ instead of $50 \%)$. With this workflow, we increased by more than threefold the amount of starting material injected (79\%). $20 \mathrm{~S}$ proteasomes were incubated alone or with a 2 -fold molar excess of PA28, with final concentrations of 0.4 and $0.8 \mu \mathrm{M}$, respectively. $5.7 \mu \mathrm{L}$ of protein were aspirated and $5.2 \mu \mathrm{L}$ were diluted in $98.8 \mu \mathrm{L}$ of protonated (peptide mapping) or deuterated buffer (20 mM Tris $\mathrm{pH} / \mathrm{pD} 7.4,1 \mathrm{mM}$ EDTA, $1 \mathrm{mM} \mathrm{DTT})$ and incubated at $20^{\circ} \mathrm{C}$ for $0,0.5,1,5,10$, and $30 \mathrm{~min}$. The final $\mathrm{D}_{2} \mathrm{O}$ percentage was $95 \%$ in the labelled samples. $99 \mu \mathrm{L}$ were then transferred to vials containing $11 \mu \mathrm{L}$ of precooled quenching solution ( $500 \mathrm{mM}$ glycine at $\mathrm{pH} 2.3)$. For experiments involving PA28 $\beta$, the quenching buffer was supplemented with $250 \mathrm{mM}$ tris- $(2-$ carboxyethyl) phosphine (TCEP) in order to reduce the disulphide bridge between Cys 21 of chain $\alpha$ and Cys 3 of chain $\beta$. After $30 \mathrm{~s}$. of quenching, $105 \mu \mathrm{L}$ were injected into a $100 \mu \mathrm{L}$ loop. Proteins were digested online with a $2.1 \times 30 \mathrm{~mm}$ Poros Immobilized Pepsin column (Life Technologies/Applied Biosystems, Carlsbad, CA, USA). The temperature of the digestion room was set at $15^{\circ} \mathrm{C}$

Chromatographic run: In order to cope with the unusual sample heterogeneity, the runtime of the chromatographic separation (12 min) was doubled compared to the one used for smaller protein complexes $(6 \mathrm{~min})$. Peptides were desalted for 3 min on a C18 pre-column (Acquity UPLC BEH $1.7 \mu \mathrm{m}$, VANGUARD) and separated on a C18 column (Acquity UPLC BEH $1.7 \mu \mathrm{m}, 1.0 \times 100 \mathrm{~mm}$ ) by the following gradient: $5-35 \%$ buffer B (100\% acetonitrile, $0.2 \%$ formic acid) for 12 $\mathrm{min}, 35-40 \%$ for $1 \mathrm{~min}, 40-95 \%$ for $1 \mathrm{~min}, 2 \mathrm{~min}$ at $95 \%$ followed by 2 cycles of $5-95 \%$ for $2 \mathrm{~min}$ and a final equilibration at 5\% buffer A (5\% acetonitrile, $0.2 \%$ formic acid) for $2 \mathrm{~min}$. The total runtime was $25 \mathrm{~min}$. The temperature of the chromatographic module was set at $4{ }^{\circ} \mathrm{C}$. Experiments were run in triplicates and the protonated buffer was injected between each triplicate to wash the column and avoid cross-over contamination.

MS acquisition: The acquisitions were performed in positive and resolution mode in the $\mathrm{m} / \mathrm{z}$ range $50-2000 \mathrm{Th}$. The sample cone and capillary voltages were set at 30 and $3 \mathrm{kV}$, respectively. The analysis cycles for nondeuterated samples alternated between a $0.3 \mathrm{~s}$ low energy scan (Trap and Transfer collision energies set to $4 \mathrm{~V}$ and $2 \mathrm{~V}$, respectively), a $0.3 \mathrm{sec}$ high energy scan (Ramp Trap and Transfer collision energies set to 18 to $40 \mathrm{~V}$ and 2 to $2 \mathrm{~V}$, respectively) and a $0.3 \mathrm{sec}$ lockspray scan $(0.1 \mu \mathrm{M}$ [Glu1]-Fibrinopeptide in $50 \%$ acetonitrile, $50 \%$ water and $0.2 \%$ formic acid infused at $10 \mu \mathrm{L} / \mathrm{min}$ ). The lockspray trap collision energy was set at $32 \mathrm{~V}$ and a GFP scan of $0.3 \mathrm{sec}$ is acquired every min. In order to double the signal intensity of deuterated peptides, deuterated samples were acquired only with the low energy and lockspray functions.

Data analysis: Peptide identification was performed with ProteinLynx Global SERVER v.3.0.2 (PLGS, Waters, Manchester, UK) based on the $\mathrm{MS}^{\mathrm{E}}$ data acquired on the nondeuterated samples. The MSMS spectra were searched against a homemade database containing sequences from the 17 std20S and i20S subunits, PA28a, PA28 $\beta$, PA28 $\gamma$, and pepsin from Sus scrofa. Peptides were filtered in DynamX v.3.0 from Waters Scientific (Manchester, UK) with the following parameters: peptides identified in at least two replicates, 0.2 fragments per amino-acid, intensity threshold 1000. The quantitative analysis of deuteration kinetics was performed using the statistical package R (R Development Core Team, 2012; http://www.Rproject.org/) on the corresponding MS intensities. The deuterium uptakes of each 
ion for each timepoint were calculated based on the theoretical maximum, considering that all amino-acids (except proline residues and the first amino-acid or each peptide) were deuterated, and then averaged (weight $=$ intensity) to get a value of relative deuterium uptake (RDU) per peptide sequence/condition/ timepoint (see Supplementary Data 4, 5, 12, 14,16). The RDUs were not corrected for back exchange. To identify the protein regions that presented conformational changes in complex vs. alone, we performed an ANOVA (Anova(), type $=$ III, singular.ok $=\mathrm{T}$ ) followed by Benjamini-Hochberg correction of the $P$ value on all quantified peptides. For each comparison, we considered significantly regulated the peptides with a corrected $P$ value $\leq 0.01$ and an absolute difference of RDU above $1.57 \%$ (four times the mean absolute difference of RDU in the entire dataset) for three successive time points (Supplementary Data 9-10). The corresponding volcano plots are presented in Supplementary Data 3, 7, 13 and all the regions statistically regulated in all the differential HDX-MS analysis are listed in Supplementary Data 8 . The RDU and differences of RDU (for protein alones or comparison between conditions, respectively) were consolidated (RDU mapping from peptide-level to protein sequence) using the values of the smallest peptide to increase the spatial resolution (see consolidation heatmaps in Supplementary Data $1-3,6,11$ ). These data (per timepoint or sum of all time points) were then used for structural data mining with the recently developed open-source web application called HDX-Viewer [https://masstools.ipbs.fr/hdx-viewer] ${ }^{67}$ that allows to directly plot and visualize the whole dataset (14 different subunits per proteasome core particle) on the proteasome 3D structure. The scripts corresponding to the quality control and statistical analysis of this dataset are available at zenodo.org [https://zenodo.org] with the https://doi.org/10.5281/ zenodo.3769174 under the Creative Commons Attribution 4.0 International licence. All molecular representations were generated in UCSF ChimeraX v.0.9 (2019-06-06) ${ }^{68}$

Modeling: The PA28 $\gamma$ model was generated using the structure of human PA28a (PDB:1AV0) as a template with the SWISS-MODEL server ${ }^{52}$. This model misses the first 3 and the last 7 amino-acids, as well as the 64-106 stretch.

Reporting summary. Further information on research design is available in the Nature Research Reporting Summary linked to this article.

\section{Data availability}

The mass spectrometry proteomics data have been deposited to the ProteomeXchange Consortium via the PRIDE ${ }^{69}$ partner repository with the dataset identifier PXD018921. All other data are available with this paper online.

\section{Code availability}

The R scripts of the analysis, with the fasta files and output tables and cxc files are freely available at zenodo.org [https://zenodo.org] with the https://doi.org/10.5281/ zenodo.3769174 under the Creative Commons Attribution 4.0 International licence.

Received: 15 May 2020; Accepted: 6 November 2020;

Published online: 01 December 2020

\section{References}

1. Collins, G. A. \& Goldberg, A. L. The logic of the 26S proteasome. Cell 169, 792-806 (2017).

2. Bard, J. A. M. et al. Structure and function of the $26 \mathrm{~S}$ proteasome. Annu. Rev. Biochem. 87, 697-724 (2018)

3. Groettrup, M., Kirk, C. J. \& Basler, M. Proteasomes in immune cells: more than peptide producers? Nat. Rev. Immunol. 10, 73-78 (2010).

4. Aiken, C. T., Kaake, R. M., Wang, X. \& Huang, L. Oxidative stress-mediated regulation of proteasome complexes. Mol. Cell. Proteomics https://doi.org/ 10.1074/mcp.M110.006924 (2011).

5. Boisvert, F.-M., Lam, Y. W., Lamont, D. \& Lamond, A. I. A quantitative proteomics analysis of subcellular proteome localization and changes induced by DNA damage. Mol. Cell. Proteomics 9, 457-470 (2010).

6. Gomes, A. V. Genetics of proteasome diseases. Scientifica 2013, 637629 (2013).

7. Coux, O., Zieba, B. A. \& Meiners, S. in Proteostasis and Disease: From Basic Mechanisms to Clinics (eds Barrio, R., Sutherland, J. D. \& Rodriguez, M. S.) 55-100 (Springer International Publishing, 2020).

8. Basler, M., Mundt, S., Bitzer, A., Schmidt, C. \& Groettrup, M. The immunoproteasome: a novel drug target for autoimmune diseases. Clin. Exp. Rheumatol. 33, S74-S79 (2015).

9. Moscovitz, O. et al. The Parkinson's-associated protein DJ-1 regulates the $20 \mathrm{~S}$ proteasome. Nat. Commun. 6, 6609 (2015)

10. Moscovitz, O. et al. A mutually inhibitory feedback loop between the $20 \mathrm{~S}$ proteasome and its regulator, NQO1. Mol. Cell 47, 76-86 (2012).
11. Bousquet-Dubouch, M.-P. et al. Affinity purification strategy to capture human endogenous proteasome complexes diversity and to identify proteasome-interacting proteins. Mol. Cell. Proteom. 8, 1150-1164 (2009).

12. Le Tallec, B. et al. 20S proteasome assembly is orchestrated by two distinct pairs of chaperones in yeast and in mammals. Mol. Cell 27, 660-674 (2007)

13. Le Tallec, B., Barrault, M.-B., Guérois, R., Carré, T. \& Peyroche, A. Hsm3/S5b participates in the assembly pathway of the $19 \mathrm{~S}$ regulatory particle of the proteasome. Mol. Cell 33, 389-399 (2009).

14. Xie, Y. Structure, assembly and homeostatic regulation of the $26 \mathrm{~S}$ proteasome. J. Mol. Cell Biol. 2, 308-317 (2010).

15. Wang, $X$. et al. The proteasome-interacting Ecm 29 protein disassembles the $26 \mathrm{~S}$ proteasome in response to oxidative stress. J. Biol. Chem. 292 16310-16320 (2017).

16. Minis, A. et al. The proteasome regulator PI31 is required for protein homeostasis, synapse maintenance, and neuronal survival in mice. Proc. Natl Acad. Sci. USA 116, 24639-24650 (2019).

17. Bousquet-Dubouch, M. P., Fabre, B., Monsarrat, B. \& Burlet-Schiltz, O. Proteomics to study the diversity and dynamics of proteasome complexes: from fundamentals to the clinic. Expert Rev. Proteomics 8, 459-481 (2011).

18. Weissman, A. M., Shabek, N. \& Ciechanover, A. The predator becomes the prey: regulating the ubiquitin system by ubiquitylation and degradation. Nat. Rev. Mol. Cell Biol. 12, 605-620 (2011).

19. Claverol, S., Burlet-Schiltz, O., Girbal-Neuhauser, E., Gairin, J. E. \& Monsarrat, B. Mapping and structural dissection of human $20 \mathrm{~S}$ proteasome using proteomic approaches. Mol. Cell. Proteomics 1, 567-578 (2002).

20. Arrigo, A.-P., Tanaka, K., Goldberg, A. L. \& Welch, W. J. Identity of the $19 \mathrm{~S}$ 'prosome' particle with the large multifunctional protease complex of mammalian cells (the proteasome). Nature 331, 192-194 (1988).

21. Cascio, P., Call, M., Petre, B. M., Walz, T. \& Goldberg, A. L. Properties of the hybrid form of the $26 \mathrm{~S}$ proteasome containing both $19 \mathrm{~S}$ and PA28 complexes. EMBO J. 21, 2636-2645 (2002)

22. Ortega, J. et al. The axial channel of the $20 \mathrm{~S}$ proteasome opens upon binding of the PA200 activator. J. Mol. Biol. 346, 1221-1227 (2005).

23. Huang, X., Luan, B., Wu, J. \& Shi, Y. An atomic structure of the human $26 \mathrm{~S}$ proteasome. Nat. Struct. Mol. Biol. 23, 778-785 (2016).

24. Xie, S. C. et al. The structure of the PA28-20S proteasome complex from Plasmodium falciparum and implications for proteostasis. Nat. Microbiol. https://doi.org/10.1038/s41564-019-0524-4 (2019).

25. Toste Rêgo, A. \& da Fonseca, P. C. A. Characterization of fully recombinant human 20S and 20S-PA200 proteasome complexes. Mol. Cell https://doi.org/ 10.1016/j.molcel.2019.07.014 (2019).

26. Guan, H. et al. Cryo-EM structures of the human PA200 and PA200-20S complex reveal regulation of proteasome gate opening and two PA200 apertures. PLoS Biol. 18, e3000654 (2020).

27. Schweitzer, A. et al. Structure of the human $26 \mathrm{~S}$ proteasome at a resolution of $3.9 \backslash$ AA. Proc. Natl Acad. Sci. USA 113, 7816-7821 (2016).

28. Wehmer, M. et al. Structural insights into the functional cycle of the ATPase module of the 26S proteasome. Proc. Natl Acad. Sci. USA 114, 1305-1310 (2017).

29. Beck, F. et al. Near-atomic resolution structural model of the yeast $26 \mathrm{~S}$ proteasome. Proc. Natl Acad. Sci. USA 109, 14870-14875 (2012).

30. Aufderheide, A. et al. Structural characterization of the interaction of Ubp6 with the 26S proteasome. Proc. Natl Acad. Sci. USA 112, 8626-8631 (2015).

31. Huber, E. M. et al. Immuno- and constitutive proteasome crystal structures reveal differences in substrate and inhibitor specificity. Cell 148, 727-738 (2012).

32. Knowlton, J. R. et al. Structure of the proteasome activator REGalpha (PA28alpha). Nature 390, 639-644 (1997).

33. Huber, E. M. \& Groll, M. The mammalian proteasome activator PA28 forms an asymmetric $\alpha 4 \beta 3$ complex. Structure 25, 1473-1480.e3 (2017).

34. Ruschak, A. M. \& Kay, L. E. Proteasome allostery as a population shift between interchanging conformers. Proc. Natl Acad. Sci. USA 109, E3454-E3462 (2012).

35. Schrader, J. et al. The inhibition mechanism of human $20 \mathrm{~S}$ proteasomes enables next-generation inhibitor design. Science 353, 594-598 (2016).

36. Ladi, E. et al. Design and evaluation of highly selective human immunoproteasome inhibitors reveal a compensatory process that preserves immune cell viability. J. Med. Chem. 62, 7032-7041 (2019).

37. Santos, R. et al. Structure of human immunoproteasome with a reversible and noncompetitive inhibitor that selectively inhibits activated lymphocytes. Nat. Commun. 8, 1-11 (2017).

38. van de Waterbeemd, M. et al. Structural analysis of a temperature-induced transition in a viral capsid probed by HDX-MS. Biophys. J. 112, 1157-1165 (2017).

39. Marcoux, J. et al. p47phox molecular activation for assembly of the neutrophil NADPH oxidase complex. J. Biol. Chem. 285, 28980-28990 (2010).

40. Guillet, V. et al. Structural insights into chaperone addiction of toxin-antitoxin systems. Nat. Commun. 10, 782 (2019). 
41. Chalmers, M. J. et al. Probing protein ligand interactions by automated hydrogen/deuterium exchange mass spectrometry. Anal. Chem. 78, 1005-1014 (2006).

42. Ugras, S. et al. Induction of the immunoproteasome subunit Lmp7 links proteostasis and immunity in $\alpha$-synuclein aggregation disorders. EBioMedicine 31, 307-319 (2018).

43. Jallow, S. et al. The presence of prolines in the flanking region of an immunodominant HIV-2 gag epitope influences the quality and quantity of the epitope generated. Eur. J. Immunol. 45, 2232-2242 (2015).

44. Pickering, A. M. \& Davies, K. J. A. Differential roles of proteasome and immunoproteasome regulators $\mathrm{Pa} 28 \alpha \beta, \mathrm{Pa} 28 \gamma$ and $\mathrm{Pa} 200$ in the degradation of oxidized proteins. Arch. Biochem. Biophys. 523, 181-190 (2012).

45. Osmulski, P. A. \& Gaczynska, M. Nanoenzymology of the 20 S proteasome: proteasomal actions are controlled by the allosteric transition. Biochemistry 41, 7047-7053 (2002).

46. Osmulski, P. A., Hochstrasser, M. \& Gaczynska, M. A tetrahedral transition state at the active sites of the $20 \mathrm{~S}$ proteasome is coupled to opening of the $a-$ ring channel. Struct. Lond. Engl. 1993, 1137-1147 (2009).

47. Huang, R., Pérez, F. \& Kay, L. E. Probing the cooperativity of Thermoplasma acidophilum proteasome core particle gating by NMR spectroscopy. Proc. Natl Acad. Sci. USA 114, E9846-E9854 (2017).

48. Park, S. et al. Reconfiguration of the proteasome during chaperone-mediated assembly. Nature 497, 512-516 (2013).

49. Arciniega, M., Beck, P., Lange, O. F., Groll, M. \& Huber, R. Differential global structural changes in the core particle of yeast and mouse proteasome induced by ligand binding. Proc. Natl Acad. Sci. USA 111, 9479-9484 (2014).

50. Whitby, F. G. et al. Structural basis for the activation of $20 \mathrm{~S}$ proteasomes by $11 \mathrm{~S}$ regulators. Nature 408, 115-120 (2000).

51. Förster, A., Masters, E. I., Whitby, F. G., Robinson, H. \& Hill, C. P. The 1.9 $\backslash$ AA structure of a proteasome-11S activator complex and implications for proteasome-PAN/PA700 interactions. Mol. Cell 18, 589-599 (2005).

52. Waterhouse, A. et al. SWISS-MODEL: homology modelling of protein structures and complexes. Nucleic Acids Res. 46, W296-W303 (2018).

53. Raule, M., Cerruti, F. \& Cascio, P. Enhanced rate of degradation of basic proteins by $26 \mathrm{~S}$ immunoproteasomes. Biochim. Biophys. Acta 1843, 1942-1947 (2014).

54. Jonik-Nowak, B. et al. PIP30/FAM192A is a novel regulator of the nuclear proteasome activator PA28\%. Proc. Natl Acad. Sci. USA 115, E6477-E6486 (2018).

55. Welk, V. et al. Inhibition of proteasome activity induces formation of alternative proteasome complexes. J. Biol. Chem. 291, 13147-13159 (2016).

56. Sheff, J. G., Hepburn, M., Yu, Y., Lees-Miller, S. P. \& Schriemer, D. C. Nanospray HX-MS configuration for structural interrogation of large protein systems. Analyst 142, 904-910 (2017).

57. Sowole, M. A., Alexopoulos, J. A., Cheng, Y.-Q., Ortega, J. \& Konermann, L. Activation of ClpP protease by ADEP antibiotics: insights from hydrogen exchange mass spectrometry. J. Mol. Biol. 425, 4508-4519 (2013).

58. Wales, T. E. \& Engen, J. R. Partial unfolding of diverse $\mathrm{SH} 3$ domains on a wide timescale. J. Mol. Biol. 357, 1592-1604 (2006).

59. Li, M. J., Guttman, M. \& Atkins, W. M. Conformational dynamics of Pglycoprotein in lipid nanodiscs and detergent micelles reveal complex motions on a wide time scale. J. Biol. Chem. 293, 6297-6307 (2018).

60. Sbardella, D. et al. The insulin-degrading enzyme is an allosteric modulator of the 20S proteasome and a potential competitor of the 19S. Cell. Mol. Life Sci. https://doi.org/10.1007/s00018-018-2807-y (2018).

61. Lesne, J., Bousquet, M.-P., Marcoux, J. \& Locard-Paulet, M. Top-down and intact protein mass spectrometry data visualization for proteoform analysis using VisioProt-MS. Bioinforma. Biol. Insights 13, 1177932219868223 (2019).

62. Stohwasser, R., Salzmann, U., Giesebrecht, J., Kloetzel, P.-M. \& Holzhütter, H.-G. Kinetic evidences for facilitation of peptide channelling by the proteasome activator PA28. Eur. J. Biochem. 267, 6221-6230 (2000).

63. Sugiyama, M. et al. Spatial arrangement and functional role of a subunits of proteasome activator PA28 in hetero-oligomeric form. Biochem. Biophys. Res. Commun. 432, 141-145 (2013).

64. Fabre, B. et al. Deciphering preferential interactions within supramolecular protein complexes: the proteasome case. Mol. Syst. Biol. 11, 771 (2015).
65. Chen, J. et al. Cryo-EM of mammalian PA28 $\alpha \beta$-iCP immunoproteasome reveals a distinct mechanism of proteasome activation by PA28a $\beta$. bioRxiv https://doi.org/10.1101/2020.04.29.067652 (2020).

66. Olshina, M. A. et al. Regulation of the $20 \mathrm{~S}$ proteasome by a novel family of inhibitory proteins. Antioxid. Redox Signal. 32, 636-655 (2020).

67. Bouyssié, D. et al. HDX-Viewer: interactive 3D visualization of hydrogendeuterium exchange data. Bioinforma. Oxf. Engl. https://doi.org/10.1093/ bioinformatics/btz550 (2019).

68. Goddard, T. D. et al. UCSF ChimeraX: meeting modern challenges in visualization and analysis. Protein Sci. Publ. Protein Soc. 27, 14-25 (2018).

69. Perez-Riverol, Y. et al. The PRIDE database and related tools and resources in 2019: improving support for quantification data. Nucleic Acids Res. 47, D442-D450 (2019).

\section{Acknowledgements}

This work was supported by the French Ministry of Research (ANR-ProteasoRegMS to J. M. and Investissements d'Avenir Program, Proteomics French Infrastructure, ANR-10INBS-08 to O.B.-S.), the 631 Fonds Européens de Développement Régional Toulouse Métropole and the Région Midi-Pyrénées (O.B.-S.), and the Novo Nordisk Foundation (NNF14CC0001).

\section{Author contributions}

All authors contributed to editing the manuscript and figures. J.L. performed the HDXMS optimization and data acquisition, model building and data analysis. J.P. performed the HDX-MS optimization, data acquisition, and data analysis. D.Z. and T.M. performed experiments. M.L.P. analyzed the data and wrote the paper. O.B.-S. provided critical input. M.P.B. provided critical expertise on the proteasome. J.M. designed, performed and supervised the experiments, data and structural analysis, and wrote the paper.

\section{Competing interests}

The authors declare no competing interests.

\section{Additional information}

Supplementary information is available for this paper at https://doi.org/10.1038/s41467020-19934-z.

Correspondence and requests for materials should be addressed to J.M

Peer review information Nature Communications thanks Frank Sobott and other, anonymous, reviewers for their contributions to the peer review of this work. Peer review reports are available.

Reprints and permission information is available at http://www.nature.com/reprints

Publisher's note Springer Nature remains neutral with regard to jurisdictional claims in published maps and institutional affiliations.

Open Access This article is licensed under a Creative Commons Attribution 4.0 International License, which permits use, sharing, adaptation, distribution and reproduction in any medium or format, as long as you give appropriate credit to the original author(s) and the source, provide a link to the Creative Commons license, and indicate if changes were made. The images or other third party material in this article are included in the article's Creative Commons license, unless indicated otherwise in a credit line to the material. If material is not included in the article's Creative Commons license and your intended use is not permitted by statutory regulation or exceeds the permitted use, you will need to obtain permission directly from the copyright holder. To view a copy of this license, visit http://creativecommons.org/ licenses/by/4.0/.

(C) The Author(s) 2020 